\title{
Genome organization and DNA accessibility control antigenic variation in trypanosomes
}

Laura S. M. Müller 1,2,3,15, Raúl O. Cosentino ${ }^{1,2,3,15}$, Konrad U. Förstner ${ }^{4,5,6}$, Julien Guizetti ${ }^{3,14}$, Carolin Wedel ${ }^{3}$, Noam Kaplan² Christian J. Janzen ${ }^{8}$, Panagiota Arampatzi ${ }^{6}$, Jörg Vogel ${ }^{9,10}$, Sascha Steinbiss ${ }^{11}$, Thomas D. Otto ${ }^{11,12}$, Antoine-Emmanuel Saliba ${ }^{9}$, Robert P. Sebra ${ }^{13}$ \& T. Nicolai Siegel ${ }^{1,2,3 *}$

Many evolutionarily distant pathogenic organisms have evolved similar survival strategies to evade the immune responses of their hosts. These include antigenic variation, through which an infecting organism prevents clearance by periodically altering the identity of proteins that are visible to the immune system of the host ${ }^{1}$. Antigenic variation requires large reservoirs of immunologically diverse antigen genes, which are often generated through homologous recombination, as well as mechanisms to ensure the expression of one or very few antigens at any given time. Both homologous recombination and gene expression are affected by threedimensional genome architecture and local DNA accessibility ${ }^{2,3}$. Factors that link three-dimensional genome architecture, local chromatin conformation and antigenic variation have, to our knowledge, not yet been identified in any organism. One of the major obstacles to studying the role of genome architecture in antigenic variation has been the highly repetitive nature and heterozygosity of antigen-gene arrays, which has precluded complete genome assembly in many pathogens. Here we report the de novo haplotypespecific assembly and scaffolding of the long antigen-gene arrays of the model protozoan parasite Trypanosoma brucei, using longread sequencing technology and conserved features of chromosome folding ${ }^{4}$. Genome-wide chromosome conformation capture (Hi-C) reveals a distinct partitioning of the genome, with antigen-encoding subtelomeric regions that are folded into distinct, highly compact compartments. In addition, we performed a range of analyses$\mathrm{Hi}-\mathrm{C}$, fluorescence in situ hybridization, assays for transposaseaccessible chromatin using sequencing and single-cell RNA sequencing - that showed that deletion of the histone variants $\mathrm{H3}$. V and H4.V increases antigen-gene clustering, DNA accessibility across sites of antigen expression and switching of the expressed antigen isoform, via homologous recombination. Our analyses identify histone variants as a molecular link between global genome architecture, local chromatin conformation and antigenic variation.

Genome sequences of several pathogens have revealed a partitioning of chromosomes, with housekeeping genes often being located in the central core and antigen genes being located in subtelomeric regions $^{5,6}$. These assemblies suggest that the linear organization of the genome may be important for restricting high levels of recombination to regions that code for antigens and for ensuring that all but one antigen is repressed.

Recently, genome-wide $\mathrm{Hi}-\mathrm{C}$ analyses have begun to uncover the $3 \mathrm{D}$ organization of chromosomes at high resolution ${ }^{4}$, which has highlighted the critical role of spatial organization and compartmentalization of DNA in the regulation of gene expression and recombination ${ }^{2,3}$.
In addition, microscopy-based analyses of the unicellular eukaryotic parasites Plasmodium falciparum and T. brucei have indicated that nuclear organization may be important for the mutually exclusive expression of antigens ${ }^{7-9}$. However, to our knowledge, the proteins that are involved in shaping genome architecture and controlling antigen expression have not yet been identified in any organism.

This study aimed to identify the process that restricts antigen expression. Specifically, we sought to identify proteins that are important for maintaining genome architecture and to determine whether global and/or local changes in chromatin conformation affect antigen expression.

In T. brucei-which is the causative agent of human sleeping sickness - the key antigens are the variant surface glycoproteins (VSGs). Most VSG genes-of which there are about 2,500-are found in long subtelomeric arrays of megabase chromosomes ${ }^{6}$. In addition, about 65 VSG genes are located on mini-chromosomes (50-150 kb in length $)^{10}$ and a smaller subset of VSG genes is located in distinct telomere-proximal polycistronic transcription units, called expression sites $^{11}$. Expression sites are grouped into metacyclic-form and bloodstream-form expression sites (MESs and BESs, respectively) on the basis of the life-cycle stage during which they can be activated. VSG genes are transcribed only when they are located within an expression site and only one of about 15 BESs is transcribed at a time, which ensures that the expression of VSG genes is mutually exclusive ${ }^{11}$. Therefore, a genome sequence that contains both subtelomeric VSG gene arrays and telomeric expression sites, which is lacking in the available T. brucei genome (isolate TREU 927) ${ }^{6}$, is required to elucidate the molecular link between genome architecture and antigenic variation.

Using PacBio single-molecule real-time (SMRT) sequencing technology, we generated an approximately 100-fold genome-sequence coverage of the T. brucei 427 Lister isolate (the most commonly used laboratory isolate) and assembled the reads into megabase chromosomes, of which there are 11 (96 contigs, Fig. 1, Extended Data Table 1). To order and orient contigs without relying on scaffolds of related parasite isolates (which may have undergone genome rearrangements), we took advantage of two ubiquitous features of chromosome organization: a distance-dependent decay of DNA-DNA interaction frequency and substantially higher interaction frequencies between DNA loci located on the same chromosome, compared to those on different chromosomes ${ }^{4}$. The high degree of subtelomeric heterozygosity enabled us to assemble the complete T. brucei genome with phased diploid subtelomeric regions (Extended Data Figs. 1, 2, Supplementary Data). In addition, RNA sequencing (RNA-seq) revealed a notable partitioning of the genome into a transcribed homozygous core and non-transcribed

\footnotetext{
${ }^{1}$ Department of Veterinary Sciences, Experimental Parasitology, Ludwig-Maximilians-Universität München, Munich, Germany. ${ }^{2}$ Biomedical Center Munich, Department of Physiological Chemistry, Ludwig-Maximilians-Universität München, Planegg-Martinsried, Germany. ${ }^{3}$ Research Center for Infectious Diseases, University of Würzburg, Würzburg, Germany. ${ }^{4} Z B$ MED - Information Centre for Life Sciences, Cologne, Germany. ${ }^{5} \mathrm{TH}$ Köln, Faculty of Information Science and Communication Studies, Cologne, Germany. ${ }^{6}$ Core Unit Systems Medicine, Institute of Molecular Infection Biology, University of Würzburg, Würzburg, Germany. ${ }^{7}$ Department of Physiology, Biophysics \& Systems Biology, Rappaport Faculty of Medicine, Technion Israel Institute of Technology, Haifa, Israel. ${ }^{8}$ Department of Cell \& Developmental Biology, Biocenter, University of Würzburg, Würzburg, Germany. ${ }^{9} \mathrm{Helmh}$ holtz Institute for RNA-based Infection Research, Würzburg, Germany. ${ }^{10}$ RNA Biology Group, Institute of Molecular Infection Biology, University of Würzburg, Würzburg, Germany. ${ }^{11}$ Wellcome Trust Sanger Institute, Hinxton, Cambridge, UK. ${ }^{12}$ Centre of Immunobiology, Institute of Infection, Immunity \& Inflammation, College of Medical, Veterinary and Life Sciences, University of Glasgow, Glasgow, UK. ${ }^{13}$ Icahn Institute and Department of Genetics and Genomic Sciences, Icahn School of Medicine at Mount Sinai, New York, NY, USA. ${ }^{14}$ Present address: Centre for Infectious Diseases, Parasitology, Heidelberg University Hospital, Heidelberg, Germany. ${ }^{15}$ These authors contributed equally: Laura S. M. Müller, Raúl O. Cosentino. *e-mail: n.siegel@Imu.de
} 


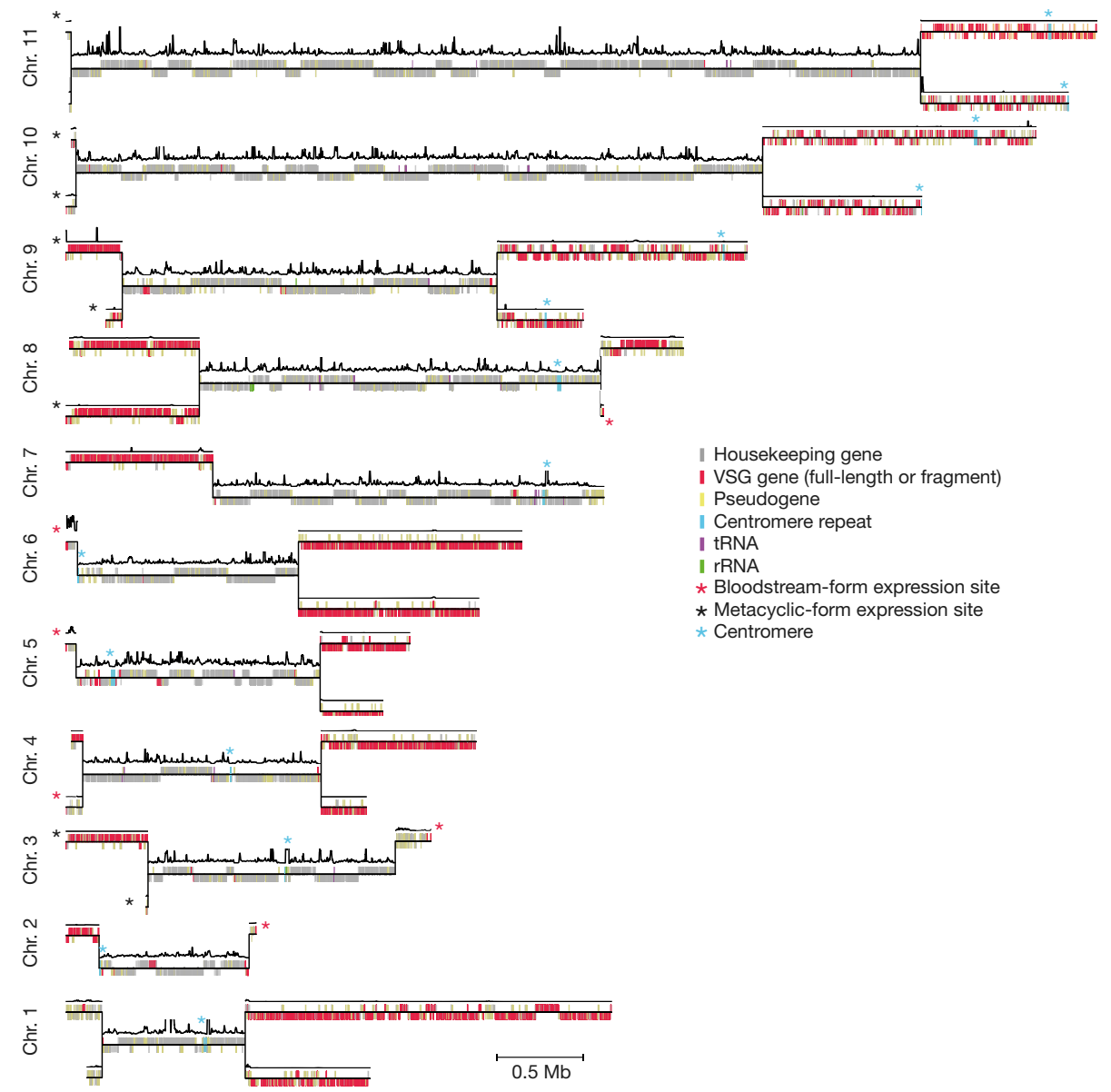

Fig. 1 | Long-read and Hi-C-based de novo assembly of the T. brucei Lister 427 genome. Only one of the two homologous chromosomes (chr.) is depicted for the homozygous chromosomal core regions $(22.71 \mathrm{Mb})$. Both chromosomes are shown for the heterozygous subtelomeric regions (19.54 Mb). Relative transcript levels (window size, 5,001 bp;

heterozygous subtelomeric regions, which encode the vast repertoire of antigens (Fig. 1).

Analysis of the frequency of intra-chromosomal DNA-DNA interaction suggested a strong compartmentalization of the T. brucei genome: centromeres and junctions between the core and subtelomeres function as the most prominent boundaries of DNA compartments. In addition, the frequency of DNA-DNA contact was substantially higher across subtelomeric regions compared to core regions, which indicates that subtelomeres are more compact than the core region (Fig. 2a, Extended Data Fig. 3). Therefore, the partitioning of the genome into transcribed housekeeping genes and non-transcribed antigen genes that is observed in the genome assembly and transcriptome data is mirrored by the $3 \mathrm{D}$ organization of the genome. In T. brucei, RNA polymerase II transcription can occur in the absence of canonical promoter motifs ${ }^{12,13}$. Thus, the high degree of compaction across subtelomeric regions probably prevents the spurious initiation of transcription and ensures mutually exclusive expression of a single VSG gene from a BES. In addition, BES-BES interactions were much more frequent than interactions among randomly chosen genomic loci, suggesting a clustering of BESs (Fig. 2b). Taken together, the Hi-C data suggest a distinct compartmentalization of the T. brucei nucleus.

Higher-order genome structures are established and maintained by architectural proteins such as CCCTC-binding factor (CTCF) and cohesin ${ }^{14}$. Histone variants are also enriched at many compartment boundaries ${ }^{15}$, but the role of these variants in shaping genome architecture remains unknown. Although CTCF appears to be absent in non-metazoans ${ }^{16}$, the major subunit of cohesin (SCC1) is present in T. brucei and the depletion of this subunit causes deregulation of VSG step size, $101 \mathrm{bp}$ ) are shown as a black line above each chromosome. BESs and MESs were assigned to the respective subtelomeric region if an unambiguous assignment based on DNA interaction data was possible (see Supplementary Information). Centromeres were assigned based on KKT2 ChIP-seq data ${ }^{30}$.

expression ${ }^{17}$. However, it has remained unclear whether this is a direct effect because SCC1 depletion strongly affects cell-cycle progression and growth rate, leading to rapid parasite death ${ }^{18}$.

Chromatin immunoprecipitation with sequencing (ChIP-seq) revealed that in T. brucei SCC1 is enriched across tRNA and rRNA genes, termination sites of RNA polymerase II transcription and most of the $3^{\prime}$ ends of BESs (Fig. 2c, d, Extended Data Fig. 4). This pattern of cohesin enrichment is reminiscent of its distribution in humans and yeast, in which cohesin is found at insulator and boundary elements such as tRNA genes ${ }^{19,20}$. The observed distribution of SCC1 is also similar to that of histone variants H3.V and, to a lesser extent, H4.V in T. brucei (Fig. 2d, Extended Data Fig. 4; also see ref. ${ }^{21}$ ). This raised the possibility that these two histone variants function together with SCC1 in shaping genome organization and the regulation of antigen expression.

To investigate a possible link between these histone variants, genome architecture and antigen expression, we determined the expression of VSG genes and genome architecture in $\Delta H 3 . V, \Delta H 4 . V$ and $\Delta H 3 . V \Delta H 4 . V$ cells. No cell cycle defect was observed in these cell lines (Extended Data Fig. 5).

Laboratory-adapted isolates, such as the one used here, switch their expression of VSG isoforms at very low frequency (about $10^{-6}$ per population doubling), and homogenously express VSG-2 (Fig. 3a; also see ref. ${ }^{22}$ ). Thus, an increase in heterogeneity of VSG gene expression can be caused by a loss of mutually exclusive expression of VSG genes in individual cells-that is, heterogeneity in antigen expression at the single-cell level-or an increased switching frequency in expression of VSG genes in different parasites (heterogeneity at the population level). 

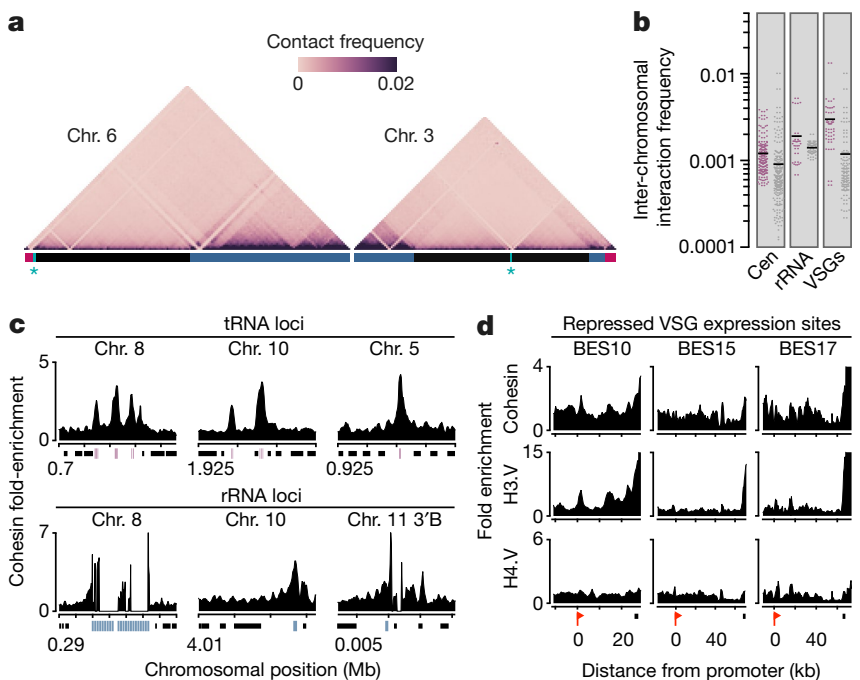

Fig. 2 Hi-C and ChIP-seq reveal partitioning of the T. brucei genome into distinct domains. a, $\mathrm{Hi}-\mathrm{C}$ heat maps of chromosomes 3 and 6 at $20-\mathrm{kb}$ resolution. Horizontal blue, black and red lines mark heterozygous subtelomeric, homozygous core regions and BESs, respectively. Centromeres are marked by asterisks. b, Scatter plot showing inter-chromosomal interaction frequencies among centromeres (cen) ( $n=206$ bins; $P=0.0029$ ), VSG genes in silent expression sites (VSGs) $\left(n=54\right.$ bins; $\left.P=1.63 \times 10^{-6}\right)$ and rRNA genes $(n=40$ bins; $P=0.0177)$ compared to a matching background sample, which was randomly selected from the interaction matrix (50-kb bin size). The background sample (grey) matches the genomic feature (red) in size and number. Selected bins with zero values were removed from both the query and background sample. $P$ values are based on Welch's $t$-test (two-sided). Black lines represent the mean. c, ChIP-seq data showing the enrichment (compared to input material) of the cohesin subunit SCC1 ( $n=3$ biologically independent experiments) across representative tRNA and rRNA genes (window size, $501 \mathrm{bp}$; step size, $101 \mathrm{bp}$ ). Black, red and blue boxes represent protein coding, tRNA and rRNA genes, respectively. Tick marks on the $x$ axis represent 5 -kb intervals. $3^{\prime} \mathrm{B}$ refers to one of the two alternative subtelomeric ends (A or B) at the $3^{\prime}$ end of chromosome 11. d, ChIP-seq data showing cohesin $(n=3$ biologically independent experiments), H3.V and H4.V (each in $n=2$ biologically independent experiments) enrichment across three transcriptionally repressed BESs (window size, 2,001 bp; step size, $501 \mathrm{bp}$ ). Red flags mark BES promoters and black boxes indicate the locations of VSG genes.

To distinguish between these possibilities and to identify the VSG genes that are expressed, we performed single-cell RNA-seq (scRNA-seq) of individual T. brucei cells. scRNA-seq data from a total of 40 wild-type and $378 \Delta H 3 . V \Delta H 4 . V$ cells revealed that-whereas all wild-type cells expressed VSG-2-in 74\% of the $\triangle H 3 . V \Delta H 4 . V$ cells, VSG-2 transcript levels contributed less than $20 \%$ of the total VSG mRNA; this indicates a switch in expression of VSG genes (Fig. 3a, Extended Data Figs. 6, 7). Activation of new VSG genes was not random, with VSG-11 being the dominant newly activated VSG gene in 230 out of 378 cells. In addition, several cells contained transcripts from multiple VSG genes, which points to a partial loss of mutually exclusive expression. To determine the stability of VSG-2 expression, we analysed $\Delta H 3 . V \Delta H 4$.V cells at two time points that were about 50 population doublings apart. Although the overall pattern remained the same (Fig. 3a, Extended Data Fig. 6), the percentage of cells that expressed only VSG-2 mRNA, or multiple VSG mRNAs, had declined by the second time point. This suggests that the process of VSG-2 deactivation had progressed further, and that the simultaneous expression of multiple VSG genes may have been a transient intermediate state. Analyses based on immunofluorescence and flow cytometry confirmed that the loss of VSG-2 mRNA resulted in a loss of VSG-2 expression (Extended Data Fig. 8). No major effect on the expression of VSG genes was observed upon deletion of H3. V or H4.V alone (Extended Data Fig. 8). a
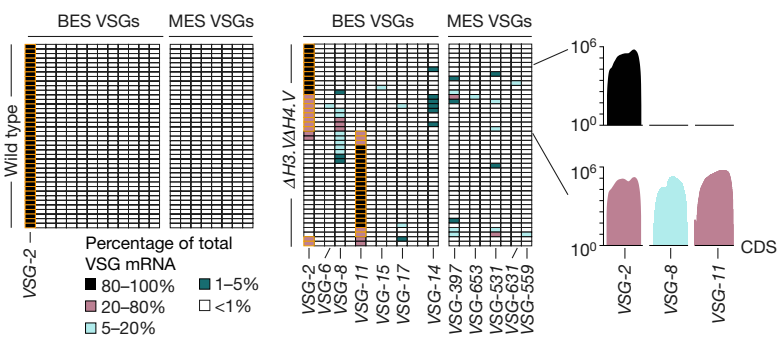

b
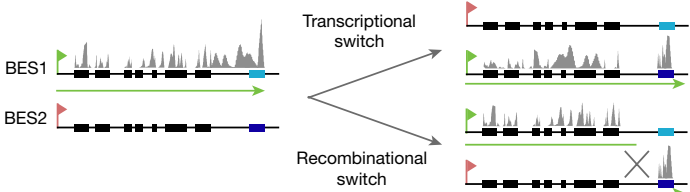

C
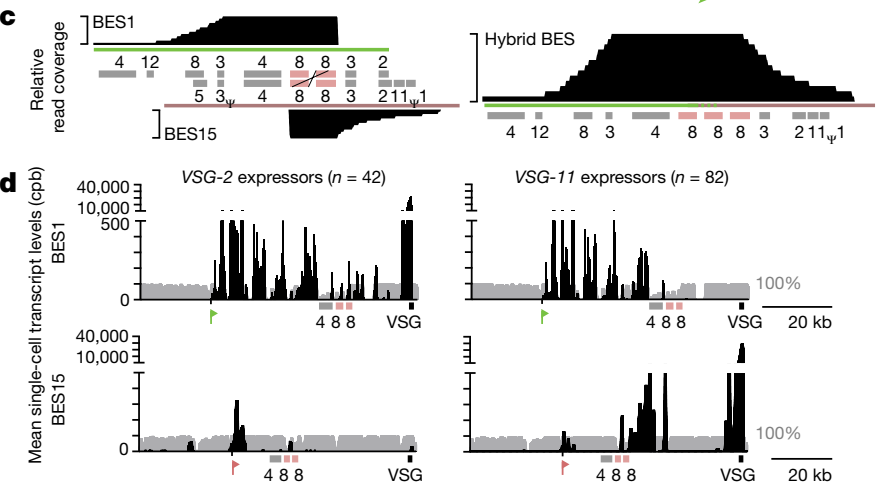

e
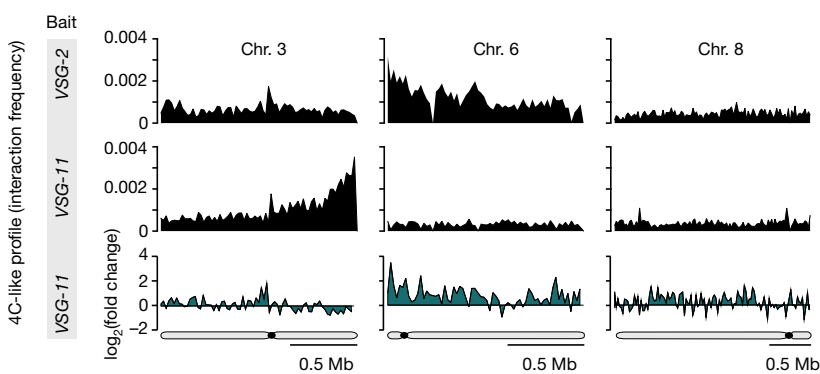

Fig. 3 Deletion of histone variants $\mathrm{H3} . \mathrm{V}$ and $\mathrm{H4} . \mathrm{V}$ leads to a switch in expression of VSG isoforms. a, scRNA-seq analysis of wild-type $(n=40)$ and $\Delta H 3 . V \Delta H 4 . V$ (first time point, $n=44$ ) cells. Each row represents data from one cell. The number of sequencing reads was normalized to account for differences in library size, gene length and uniqueness of VSG gene sequence. Only uniquely mapping reads were considered. The total number of VSG transcripts per cell is set to $100 \%$ (for details, see Methods, Extended Data Fig. 7). The colour code indicates the contribution of individual VSG transcripts to the pool of VSG transcripts in a single cell. The dominant VSG isoform is depicted with an orange border. For selected cells, the read coverage is shown across VSG-2, VSG-8 and VSG-11 (with $500 \mathrm{bp}$ of surrounding sequence). CDS, coding sequence. $\mathbf{b}$, Outline of the VSG switching mechanisms described for T. brucei. Green and red flags mark the active and repressed promoters, respectively. Green lines and grey bars indicate regions of expected transcription for the two different scenarios. c, Sequencing coverage across BES1 (left, top), BES15 (left, bottom) and a hybrid BES consisting of the $5^{\prime}$-BES1 and $3^{\prime}$-BES15 (right). Coverage is based on SMRT sequencing reads $>10 \mathrm{~kb}$ from $\triangle H 3 . V \Delta H 4 . V$ gDNA that map to BES1 and BES15. The cross represents the site of recombination. Boxes represent expression-site-associated genes and $\psi$ denotes a pseudogene. d, scRNA-seq-based analysis of $\Delta H 3 . V \Delta H 4 . V$ cells that exclusively express VSG-2 $(n=42)$ or VSG-11 $(n=82)$. The average transcript levels (counts per billion, $\mathrm{cpb}$ ) based on uniquely mapping reads across BES1 and BES15 are shown. Grey bars represent degree of uniqueness. e, $4 \mathrm{C}$-like inter-chromosomal interaction profiles (based on $\mathrm{Hi}-\mathrm{C}$-data, $20-\mathrm{kb}$ bin size) showing the average interaction frequencies of BES1 (top) and BES15 (middle) with chromosomes 3, 6 and 8 in wild-type cells and the fold change $\left(\log _{2}\right)$ in interactions of BES15 (bottom) with chromosomes 3, 6 and 8 after deletion of H3. V and H4.V. 
In T. brucei, the switching of expression of VSG genes occurs by two distinct mechanisms ${ }^{11}$ : either by switching transcription from one BES to another (transcriptional switch) or by a recombination-based event that leads to the replacement of the previously active VSG gene with a new VSG gene from a different genomic location (recombinational switch, Fig. 3b)

To gain insight into the mechanism by which histone variants affect antigen expression, we sequenced $\Delta H 3 . V \Delta H 4 . V$ genomic DNA using SMRT sequencing technology. The SMRT data indicated that, in most cells, recombination had occurred between an expressionsite-associated gene 8 (ESAG8) gene pair that was present in both BES1 and BES15. The data also revealed that the new chimeric BES contained three copies of ESAG8, one from BES1 and two from BES15 (Fig. 3c). scRNA-seq and Hi-C data support a recombination event (Fig. 3d, e). $\mathrm{Hi}-\mathrm{C}$ data revealed that, upon deletion of $H 3 . V$ and H4. $V$, the interaction frequency between VSG-11 and the $5^{\prime}$ end of chromosome 6-where VSG-2 is located in wild-type cells-increased, indicating that VSG-11 had relocated to chromosome 6.

Studies in different organisms have shown that the frequency of recombination is affected by spatial proximity and DNA accessibility ${ }^{23,24}$. Thus, to determine whether histone variants contribute to genome architecture and/or local DNA accessibility, we performed $\mathrm{Hi}-\mathrm{C}$ and assays for transposase-accessible chromatin using sequencing (ATAC-seq). Hi-C data from $\triangle H 3 . V$ cells revealed marked changes in inter-chromosomal interactions (Fig. 4a, top) and a significant increase in interactions among repressed BESs (Fig. 4b), pointing to a loss of constraints that may have 'anchored' the BESs to specific nuclear sites. In support of these $\mathrm{Hi}-\mathrm{C}$ data, fluorescence in situ hybridization (FISH) data revealed a strong clustering of telomeric repeats upon deletion of $\mathrm{H3} . \mathrm{V}$ (Fig. 4c, d). By contrast, deletion of H4.V affected genome architecture only modestly (Fig. 4a, bottom). Unlike the Hi-C data, our ATAC-seq data indicated that promoter-proximal DNA accessibility increased upon H3. $V$ or H4.V deletion (Fig. 4e). However, only $\Delta H 3 . V \Delta H 4 . V$ cells exhibited high DNA accessibility across the entire length of transcriptionally repressed BESs (Fig. 4e bottom, Extended Data Fig. 9).

In summary, the Hi-C and ATAC-seq data indicate that although deletion of H3. $V$ was responsible for the majority of genome architectural changes and increased BES clustering, this alone was not sufficient to induce a switch in expression of VSG genes. Only the concurrent deletion of $H 3$. $V$ and H4. $V$, which also strongly increased DNA accessibility across transcriptionally repressed BESs, enhanced the rate of recombination-based switching of VSG genes.

The depletion of histone $\mathrm{H} 3$ was previously shown to upregulate BES proximal-promoter activity-presumably via a general increase in DNA accessibility-but did not cause deregulation of VSG genes ${ }^{25}$. We hypothesize that the marked increase in switching frequency of VSG gene expression results from the combination of decreased spatial distance between BESs and increased local DNA accessibility (Fig. 4f).

The activation of new VSG genes did not occur at random; this non-random activation has previously been observed for infections of different hosts ${ }^{26,27}$. In a small number of cells, we detected transcripts from different VSG isoforms. This loss of mutually exclusive expression of VSG genes may be caused by increased DNA accessibility upon the deletion of histone variants, which may result in promiscuous RNA polymerase II transcription. Our observations that even in $\Delta H 3$. $V \Delta H 4 . V$ cells not all expression sites are transcribed and that specific 'pairs' of VSG genes tend to be co-expressed, suggest that there are additional constraints imposed by genome organization or VSG protein structure $^{28}$. At the genome level, co-activated VSG genes may have to be localized in close proximity to ensure sufficient levels of an activating factor $^{8,29}$; alternatively, differences in VSG protein structure may make it impossible for the parasite to tolerate certain mosaic surface coats.

In this study, we have demonstrated how evolutionarily conserved features of genome architecture can be exploited for the de novo scaffolding of phased diploid genomes. The use of Hi-C, scRNA-seq
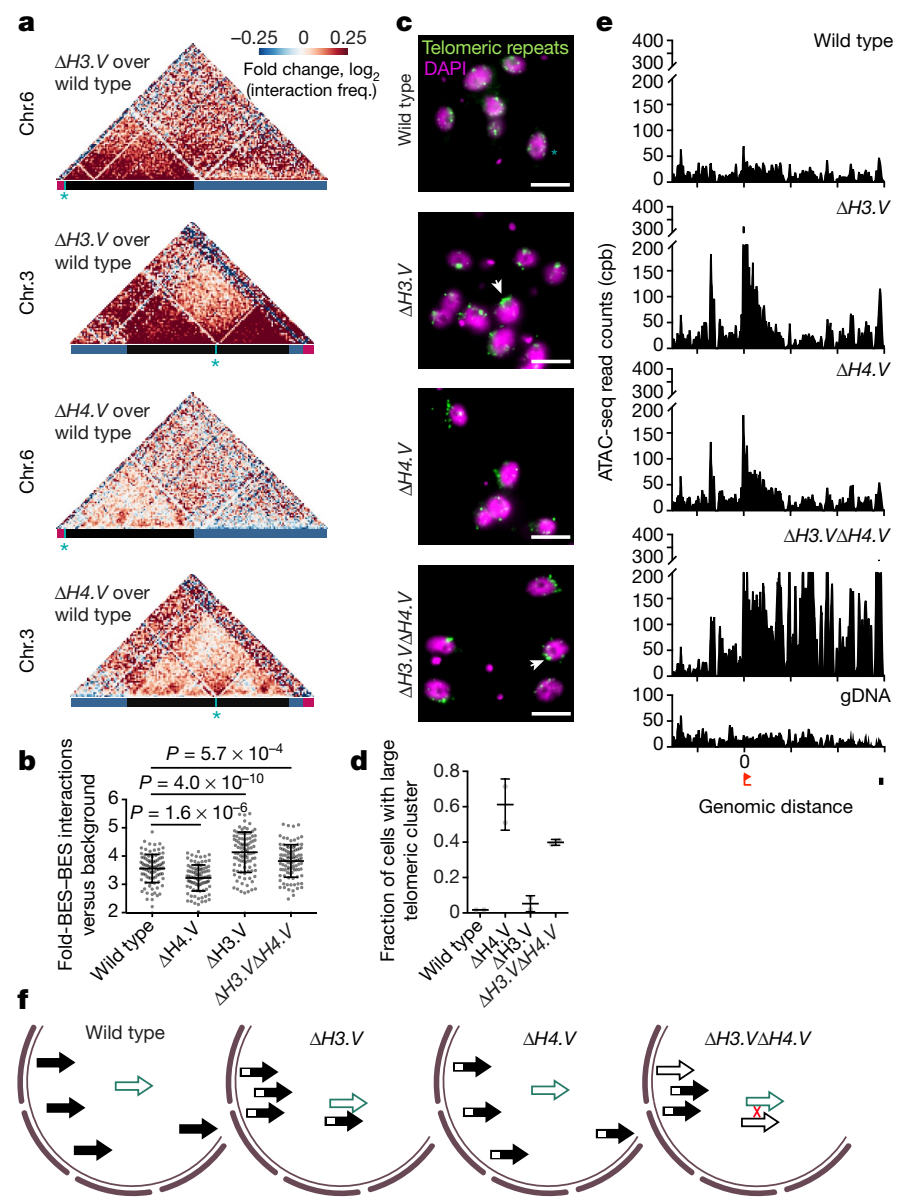

Fig. 4 | Histone variants H3.V and H4.V influence global and local chromatin structures. a, Hi-C heat map showing the fold change $\left(\log _{2}\right)$ in DNA-DNA interaction frequency between wild-type and $\triangle H 3 . V$ (top two panels) and $\Delta H 4 . V$ cells (bottom two panels). b, Fold change in DNADNA interaction frequency among VSG genes located in BESs compared to background DNA-DNA interactions in wild-type, in $\Delta H 4 . V, \Delta H 3 . V$ and $\Delta H 3 . V \Delta H 4 . V$ cells. The ratios for each cell line were calculated for 100 randomly selected background regions. Mean \pm s.d. Significance was determined using Welch's $t$-test (two-sided). c, FISH with probes against telomeric repeats (Alexa Fluor488, green), $n=2$ biologically independent experiments. Scale bar, $5 \mu \mathrm{m}$. d, Quantification of telomere signal to determine the fraction of cells containing large telomeric clusters (white arrows in c) was performed using Imaris 8 and is based on the analysis of 1,128 cells. Means \pm s.d. of two replicates are shown (wild type: $n=116,221 ; \Delta H 3 . V: n=140,102 ; \Delta H 4 . V: n=146,107$ and $\Delta H 3 . V \Delta H 4 . V: n=190,106)$. e, ATAC-seq data $(n=2$ biologically independent experiments) across BES15 (repressed in wild-type cells). gDNA read coverage (bottom) is shown to illustrate mappability of reads (window size, $501 \mathrm{bp}$; step size, $101 \mathrm{bp}$ ). Red flag and black box indicate the position of the promoter and the VSG gene, respectively. Tick marks on $x$ axes represent $20-\mathrm{kb}$ intervals. $\mathbf{f}$, Model illustrating the influence of $\mathrm{H} 3 . \mathrm{V}$ and H4.V on genome architecture and local DNA accessibility. H3.V and $\mathrm{H} 4$.V single knockouts alone mediate only partial opening of BESs (half open arrow) and H3.V knockout leads to a spatial rearrangement of BESs inside the nucleus, whereas deletion of both histone variants is required to obtain the fully opened BESs (open arrow) and spatial proximity of BESs that facilitate recombination (red cross) and lead to the expression of a new VSG isoform.

and ATAC-seq-to our knowledge, all used here for the first time in T. brucei-opened opportunities for genome assembly and the characterization of the mechanism that underlies VSG switching in $\Delta H 3$. $V \Delta H 4$. $V$ cells. Our data reveal that histone variants can function as architectural proteins, and that changes in global genome architecture and local chromatin configuration can induce extensive switches in antigen expression. 


\section{Online content}

Any methods, additional references, Nature Research reporting summaries, source data, statements of data availability and associated accession codes are available at https://doi.org/10.1038/s41586-018-0619-8.

Received: 13 July 2017; Accepted: 3 September 2018; Published online 17 October 2018.

1. Deitsch, K. W., Lukehart, S. A. \& Stringer, J. R. Common strategies for antigenic variation by bacterial, fungal and protozoan pathogens. Nat. Rev. Microbiol. 7, 493-503 (2009).

2. Hager, G. L., McNally, J. G. \& Misteli, T. Transcription dynamics. Mol. Cell 35, 741-753 (2009).

3. Misteli, T. \& Soutoglou, E. The emerging role of nuclear architecture in DNA repair and genome maintenance. Nat. Rev. Mol. Cell Biol. 10, 243-254 (2009).

4. Lajoie, B. R., Dekker, J. \& Kaplan, N. The hitchhiker's guide to Hi-C analysis: practical guidelines. Methods 72, 65-75 (2015).

5. Otto, T. D. et al. Long read assemblies of geographically dispersed Plasmodium falciparum isolates reveal highly structured subtelomeres. Wellcome Open Res. 3, 52 (2018).

6. Berriman, M. et al. The genome of the African trypanosome Trypanosoma brucei. Science 309, 416-422 (2005).

7. Lopez-Rubio, J. J., Mancio-Silva, L. \& Scherf, A. Genome-wide analysis of heterochromatin associates clonally variant gene regulation with perinuclear repressive centers in malaria parasites. Cell Host Microbe 5, 179-190 (2009).

8. Chaves, I. et al. Subnuclear localization of the active variant surface glycoprotein gene expression site in Trypanosoma brucei. Proc. Natl Acad. Sci. USA 95 12328-12333 (1998)

9. Navarro, M. \& Gull, K. A pol I transcriptional body associated with VSG mono-allelic expression in Trypanosoma brucei. Nature 414, 759-763 (2001)

10. Cross, G. A., Kim, H. S. \& Wickstead, B. Capturing the variant surface glycoprotein repertoire (the VSGnome) of Trypanosoma brucei Lister 427 Mol. Biochem. Parasitol. 195, 59-73 (2014).

11. Horn, D. Antigenic variation in African trypanosomes. Mol. Biochem. Parasitol. 195, 123-129 (2014)

12. McAndrew, M., Graham, S., Hartmann, C. \& Clayton, C. Testing promoter activity in the trypanosome genome: isolation of a metacyclic-type VSG promoter, and unexpected insights into RNA polymerase II transcription. Exp. Parasitol. 90. 65-76 (1998)

13. Wedel, C., Förstner, K. U., Derr, R. \& Siegel, T. N. GT-rich promoters can drive RNA pol II transcription and deposition of H2A.Z in African trypanosomes. EMBO J. 36, 2581-2594 (2017).

14. Merkenschlager, M. \& Odom, D. T. CTCF and cohesin: linking gene regulatory elements with their targets. Cell 152, 1285-1297 (2013).

15. Millau, J. F. \& Gaudreau, L. CTCF, cohesin, and histone variants: connecting the genome. Biochem. Cell Biol. 89, 505-513 (2011).

16. Heger, P., Marin, B., Bartkuhn, M., Schierenberg, E. \& Wiehe, T. The chromatin insulator CTCF and the emergence of metazoan diversity. Proc. Natl Acad. Sci. USA 109, 17507-17512 (2012).

17. Landeira, D., Bart, J. M., Van Tyne, D. \& Navarro, M. Cohesin regulates VSG monoallelic expression in trypanosomes. J. Cell Biol. 186, 243-254 (2009).

18. Gluenz, E., Sharma, R., Carrington, M. \& Gull, K. Functional characterization of cohesin subunit SCC1 in Trypanosoma brucei and dissection of mutant phenotypes in two life cycle stages. Mol. Microbiol. 69, 666-680 (2008).

19. Raab, J. R. \& Kamakaka, R. T. Insulators and promoters: closer than we think. Nat. Rev. Genet. 11, 439-446 (2010).

20. Van Bortle, K. \& Corces, V. G. Nuclear organization and genome function. Annu. Rev. Cell Dev. Biol. 28, 163-187 (2012).

21. Siegel, T. N. et al. Four histone variants mark the boundaries of polycistronic transcription units in Trypanosoma brucei. Genes Dev. 23, 1063-1076 (2009)

22. Horn, D. \& Cross, G. A. Analysis of Trypanosoma brucei vsg expression site switching in vitro. Mol. Biochem. Parasitol. 84, 189-201 (1997).

23. Roukos, V. et al. Spatial dynamics of chromosome translocations in living cells. Science 341, 660-664 (2013)

24. Hogenbirk, M. A. et al. Defining chromosomal translocation risks in cancer. Proc. Natl Acad. Sci. USA 113, E3649-E3656 (2016).

25. Alsford, S. \& Horn, D. Cell-cycle-regulated control of VSG expression site silencing by histones and histone chaperones ASF1A and CAF-1b in Trypanosoma brucei. Nucleic Acids Res. 40, 10150-10160 (2012).
26. Barry, J. D. \& McCulloch, R. in Advances in Parasitology, Vol. 49 (eds Baker, J. R. et al.) 1-70 (Academic Press, London, 2001).

27. Mugnier, M. R., Cross, G. A. \& Papavasiliou, F. N. The in vivo dynamics of antigenic variation in Trypanosoma brucei. Science 347, 1470-1473 (2015).

28. Pinger, J. et al. African trypanosomes evade immune clearance by O-glycosylation of the VSG surface coat. Nat. Microbiol. 3, 932-938 (2018).

29. Glover, L., Hutchinson, S., Alsford, S. \& Horn, D. VEX1 controls the allelic exclusion required for antigenic variation in trypanosomes. Proc. Natl Acad. Sci. USA 113, 7225-7230 (2016).

30. Akiyoshi, B. \& Gull, K. Discovery of unconventional kinetochores in kinetoplastids. Cell 156, 1247-1258 (2014).

Acknowledgements We thank S. Gorski and members of the Siegel laboratory for critical reading of the manuscript. We thank T. Achmedov for scRNA-seq technical assistance, M. Berriman, G. Ramasamy, P. Myler and L. Barquist for assistance with the genome assembly, J. Dekker, M. Imakaev, J. M. Belton and B. R. Lajoie for advice on Hi-C experimental design and analysis, K. Ersfeld for advice on epitope tagging of SCC1 and FISH, S. Kirchner and A. R. Batista for suggestions on ATAC-seq, T. Straub and F. Goth for providing server space and all members of the Engstler, Janzen, Kramer, Morriswood and Ladurner laboratories for valuable discussions. We thank $\mathrm{C}$. Clayton and L. Glover for reagents and M. Urbiniak for sharing unpublished SCC1 data. This work was funded by the Young Investigator Program of the Research Center for Infectious Diseases (ZINF) at the University of Würzburg, Germany, the German Research Foundation (SI 1610/2-1 and SI 1610/3-1), the Center for Integrative Protein Science (CIPSM) and by an ERC Starting Grant (3D_Tryps 715466). L.S.M.M. was supported by a grant of the German Excellence Initiative to the Graduate School of Life Science, University of Würzburg. R.O.C. was supported by a Georg Forster Fellowship (Humboldt Foundation). T.D.O. was funded by Wellcome Trust grant: 098051.

Reviewer information Nature thanks F. Tang, C.-L. Wei and the other anonymous reviewer(s) for their contribution to the peer review of this work.

Author contributions Experiments were designed by T.N.S. and L.S.M.M. and carried out by L.S.M.M. unless indicated otherwise. scRNA-seq was performed by P.A., L.S.M.M. and A.-E.S. with the assistance of J.V. N.K. provided advice on $\mathrm{Hi}-\mathrm{C}$ experiments, data analysis and the genome assembly. J.G. performed FISH. C.W. performed ATAC-seq. Genome assembly was done by R.O.C. and R.P.S. The quality of the assembly was assessed by T.D.O. and S.S. K.U.F. and L.S.M.M. performed computational analyses of Hi-C data and R.O.C. did the same for the RNA-seq and scRNA-seq data. C.J.J. performed flow cytometry. T.N.S., L.S.M.M. and R.O.C. wrote the manuscript.

Competing interests The authors declare no competing interests.

Additional information

Extended data is available for this paper at https://doi.org/10.1038/s41586018-0619-8.

Supplementary information is available for this paper at https://doi.org/ 10.1038/s41586-018-0619-8.

Reprints and permissions information is available at http://www.nature.com/ reprints.

Correspondence and requests for materials should be addressed to T.N.S. Publisher's note: Springer Nature remains neutral with regard to jurisdictional claims in published maps and institutional affiliations.

Open Access This article is licensed under a Creative Commons Attribution 4.0 International License, which permits use, sharing, adaptation, distribution and reproduction in any medium or format, as long as you give appropriate credit to the original author(s) and the source, provide a link to the Creative Commons license, and indicate if changes were made. The images or other third party material in this article are included in the article's Creative Commons license, unless indicated otherwise in a credit line to the material. If material is not included in the article's Creative Commons license and your intended use is not permitted by statutory regulation or exceeds the permitted use, you will need to obtain permission directly from the copyright holder. To view a copy of this license, visit http://creativecommons.org/ licenses/by/4.0/. 


\section{METHODS}

No statistical methods were used to predetermine sample size. The experiments were not randomized and investigators were not blinded to allocation during experiments and outcome assessment.

Cell culture. All T. brucei strains used in this study are derivatives of the Lister 427 bloodstream-form isolate. Cells were cultured in HMI-11 medium (HMI-9 ${ }^{31}$ without serum plus) at $37^{\circ} \mathrm{C}$ up to a density of $10^{6} \mathrm{cells} / \mathrm{ml}$. If required, drugs were used at standard concentrations.

Cell lines. $\Delta H 3 . V$ and $\Delta H 4 . V$ cells used in this study have previously been published $^{21,32}$. After generation of a transgenic cell line, the correct tagging of a gene or the deletion of gene was verified by PCR. Cell lines were not tested for mycoplasma contamination.

Ty1-H3.V/DH3.V cells. To delete the first H3.V allele (Tb927.10.15350), the regions upstream and downstream of the H3.V CDS were PCR-amplified using the following primer pairs: H3.V_01_F, H3.V_02_R and H3.V_03_F, H3.V_04_R (see Supplementary Table 4 for a full list of oligonucleotides) and cloned into pyrFEKO-Puro ${ }^{33}$ using InFusion HD Cloning Plus reagents (Clontech) at PvuII/ HindIII and BamHI/SbfI restriction sites. The resulting plasmid was linearized with PvuII and SbfI and stably transfected into the H3.V locus of T. brucei wild-type cells to generate $H 3 . V / \Delta H 3 . V$ cells. To add an N-terminal $2 \times$ Tyl tag to the second H3.V allele, the sequence of 326-bp upstream of the H3.V CDS (H3.V_05_F, H3.V_06_R) was cloned into the ApaI/NotI linearized vector pPOTv3-2 $\times$ Ty1 using InFusion HD Cloning Plus reagents (Clontech). Downstream of the blasticidin resistance marker and the Ty1-tag, a 417-bp DNA sequence homologous to the H3.V CDS 5'-end (H3.V_07_F, H3.V_08_R) was amplified (leaving out the ATG start codon) and likewise inserted using SacI and NheI restriction sites. The tag sequence was subsequently replaced by a codon-optimized version: oligonucleotides containing two codon-optimized Ty1 coding sequences (H3.V_09 and H3.V_10) were annealed, digested with HindIII and SacI and ligated into the HindII/SacI-linearized plasmid. Finally, the plasmid was linearized with ApaI and NheI restriction enzymes and stably transfected into $H 3 . V / \Delta H 3$. V cells to generate Ty1-H3. V/ $\triangle H 3 . V$.

$\Delta \mathrm{H} 3 . \mathrm{V} \Delta \mathrm{H} 4 . \mathrm{V}$ double-knockout cells. To delete H4.V, the upstream (H4.V_11_F, H4.V_12_R) and downstream (H4.V_13_F, H4.V_14_R) regions flanking the H4.V CDS (Tb927.2.2670) were amplified from bloodstream-form wild-type gDNA and purified using NucleoSpin Gel and PCR Clean-up kit (Macherey-Nagel). The PCR product of the upstream region was inserted into the plasmid pyrFEKO-Neo ${ }^{33}$ using InFusion HD Cloning Plus reagents (Clontech) between HindIII and AgeI restriction sites. The downstream region was integrated by ligation using BamHI and SbfI restriction sites. The neomycin resistance cassette was exchanged with a blasticidin or a phleomycin resistance marker, respectively, using the $\mathrm{BgIII}$ and $\mathrm{XbaI}$ sites that flank the resistance marker. To this end, the blasticidin and phleomycin cassettes were excised from pyrFEKO-BSD or pyrFEKO-Phleo, purified and ligated into the target plasmid. The plasmids were linearized with NheI and SbfI and stably transfected into the previously published $\Delta H 3 . V$ cell line $\mathrm{l}^{32}$.

Ty1-SCC1/ $\triangle$ SCC1 cells. To delete the first SCC1 allele (Tb927.7.6900), the flanking regions upstream (Scc1_15_F, Scc1_16_R) and downstream (Scc1_17_F, Scc1_18_R) of the SCC1 CDS were amplified, digested with PvuII/HindIII and $\mathrm{BamHI} / \mathrm{SbfI}$, respectively, and ligated into pyrFEKO-Hyg ${ }^{33}$ at PvuII/HindIII and BamHI/SbfI restriction sites. Wild-type cells were transfected with the linearized plasmid (PvuII/SbfI) to obtain SCC1/DSCC1 cells. For the N-terminal Ty1-tagging of the second SCC1 allele, the $3^{\prime}$ end of the SCC1 5' UTR was amplified (Scc1_19_F, Scc1_20_R), digested with ApaI and NotI and ligated into the ApaI/NotI-linearized vector pPOTv3-2×Ty1. Next, the $5^{\prime}$ end of the SCC1 CDS was amplified (leaving out the ATG start codon) (Scc1_21_F, Scc1_22_R), digested with SacI and NheI and ligated into the likewise-digested plasmid. The Ty1-tag was exchanged by a codon-optimized version as described for $\mathrm{N}$-terminal tagging of $H 3 . \mathrm{V}$ (see above). The ApaI/NheI linearized plasmid was stably transfected into SCC1/ $\triangle S C C 1$ T. brucei cells to generate Ty1-SCC1/ $\triangle S C C 1$.

The cell line in which both endogenous $H 4$. V alleles are knocked out and ectopic overexpression of a Tyl-tagged version of $\mathrm{H} 4$. V can be induced has previously been published ${ }^{21}$.

In situ Hi-C. Because $\Delta H 3 . V^{32}$ and $\Delta H 4 . V^{21}$ cells had been generated in a single marker' background ${ }^{34}$, we generated the $\Delta H 3 . V \Delta H 4 . V$ cells in a single marker background and compared the Hi-C profiles of the transgenic cell lines to those generated from single marker cells. Thus, all 'wild-type' $\mathrm{Hi}-\mathrm{C}$ data shown in Figs. 2-4 and Extended Data Figs. 3, 4 are generated from single marker cells. Hi-C data from 'true' wild-type cells (Lister 427, MiTat 1.2) were also generated, but used only for the genome assembly.

In situ Hi-C was performed based on previously published protocols ${ }^{35,36}$ and adapted to T. brucei: $2 \times 10^{8}$ cells (wild type, single marker, $\Delta H 3 . V, \Delta H 4$. $V$ and $\triangle H 3 . V \Delta H 4 . V$ ) were collected and resuspended in $40 \mathrm{ml}$ of $1 \times$ trypanosome dilution buffer $\left(1 \times\right.$ TDB; $0.005 \mathrm{M} \mathrm{KCl}, 0.08 \mathrm{M} \mathrm{NaCl}, 0.001 \mathrm{M} \mathrm{MgSO}_{4} \times 7 \mathrm{H}_{2} \mathrm{O}, 0.02$
$\mathrm{M} \mathrm{Na}_{2} \mathrm{HPO}_{4}, 0.002 \mathrm{M} \mathrm{Na}_{2} \mathrm{HPO}_{4} \times 2 \mathrm{H}_{2} \mathrm{O}, 0.02 \mathrm{M}$ glucose). Cells were fixed in the presence of $1 \%$ formaldehyde for $20 \mathrm{~min}$ at room temperature by addition of $4 \mathrm{ml}$ of formaldehyde solution (50 mM Hepes- $\mathrm{KOH} \mathrm{pH} \mathrm{7.5,} 100 \mathrm{mM} \mathrm{NaCl}, 1 \mathrm{mM}$ EDTA pH 8.0, 0.5 mM EGTA pH 8.0, 11\% formaldehyde). The reaction was stopped by addition of $3 \mathrm{ml}$ of $2 \mathrm{M}$ glycine and incubation for $5 \mathrm{~min}$ at room temperature and 15 min on ice. Cells were washed twice in $1 \times$ TDB and the cell pellet was snapfrozen in liquid nitrogen. Cells were resuspended in $1 \mathrm{ml}$ of permeabilization buffer (100 mM KCl, $10 \mathrm{mM}$ Tris pH 8.0, 25 mM EDTA) supplemented with protease inhibitors (1.5 mM pepstatin A, $4.25 \mathrm{mM}$ leupeptin, $1.06 \mathrm{mM}$ PMSF, $1.06 \mathrm{mM}$ TLCK) and digitonin ( $200 \mu \mathrm{M}$ final concentration) and incubated for $5 \mathrm{~min}$ at room temperature. Cells were washed twice in $1 \times$ NEBuffer3.1 (NEB, B7003S) and resuspended in $342 \mu \mathrm{l}$ of $1 \times$ NEBuffer3.1. After addition of $38 \mu \mathrm{l}$ of $1 \%$ SDS, and an incubation at $65^{\circ} \mathrm{C}$ for $10 \mathrm{~min}$, the SDS was quenched by addition of $43 \mu \mathrm{l}$ of $10 \%$ Triton-X 100 (Sigma) and the incubation was continued at room temperature for $15 \mathrm{~min}$. Another $35 \mu \mathrm{l}$ of water, $13 \mu \mathrm{l}$ of $10 \times$ NEBuffer3.1 and 100 units of MboI (NEB, R0147M) were added and the chromatin was digested at $37^{\circ} \mathrm{C}$ overnight while shaking. To inactivate MboI, the sample was incubated at $65^{\circ} \mathrm{C}$ for $20 \mathrm{~min}$. Restriction fragments were biotinylated by supplementing the reaction with $60 \mu \mathrm{l}$ of fill-in mix (0.25 mM biotin-14-dATP (Life Technologies, 19524016), $0.25 \mathrm{mM}$ dCTP, $0.25 \mathrm{mM}$ dGTP, $0.25 \mathrm{mM}$ dTTP (Fermentas), $40 \mathrm{U}$ of DNA polymerase I, large (Klenow) fragment (NEB, M0210)) and incubation at $23^{\circ} \mathrm{C}$ for $4 \mathrm{~h}$. The end-repaired chromatin was transferred to $665 \mu \mathrm{l}$ of ligation mix (1.8\% Triton-X 100, $0.18 \mathrm{mg}$ BSA, $1.8 \times$ T4 DNA Ligase Buffer (Invitrogen, 46300018) and $5 \mu \mathrm{l}$ of T4 DNA ligase (invitrogen, 15224025) were added. The ligation was performed for $4 \mathrm{~h}$ at $16^{\circ} \mathrm{C}$ with interval shake. Crosslinks were reversed by adding $50 \mu \mathrm{l}$ of $10 \mathrm{mg} / \mathrm{ml}$ proteinase $\mathrm{K}\left(65^{\circ} \mathrm{C}\right.$ for $\left.4 \mathrm{~h}\right)$ and another addition of $50 \mu \mathrm{l} \mathrm{of} 10 \mathrm{mg} / \mathrm{ml}$ proteinase $\mathrm{K}, 80 \mu \mathrm{l}$ of $5 \mathrm{M} \mathrm{NaCl}$ and $70 \mu \mathrm{l}$ of $10 \% \operatorname{SDS}\left(65^{\circ} \mathrm{C}\right.$, overnight).

The DNA was precipitated with ethanol and resuspended in $257 \mu \mathrm{l}$ of TLE (10 mM Tris-HCl, $0.1 \mathrm{mM}$ EDTA, $\mathrm{pH} 8.0)$. SDS was added to a final concentration of $0.1 \%$ and the sample was split among two tubes for sonication (Covaris S220; microtubes, $175 \mathrm{~W}$ peak incident power, $10 \%$ duty factor, 200 cycles per burst, $240 \mathrm{~s}$ treatment). The samples were recombined and the volume was adjusted to $300 \mu \mathrm{l}$ with TLE. Fragments between 100 and $400 \mathrm{bp}$ in size were selected using Agencourt AMPure XP beads (Beckman Coulter), according to the manufacturer's instructions. The DNA fragments were eluted off the beads in $55 \mu$ l of TLE.

For end-repair and biotin removal from unligated ends, $70 \mu \mathrm{l}$ of end-repair mix was added $(1 \times$ Ligation buffer $(\mathrm{NEB}), 357 \mu \mathrm{M}$ dNTPs, 25U T4 PNK (NEB, M0201), 7.5U T4 DNA polymerase I (NEB, M0203), 2.5U DNA polymerase I, large (Klenow) fragment (NEB, M0210)) and incubated for $30 \mathrm{~min}$ at $20^{\circ} \mathrm{C}$ and $20 \mathrm{~min}$ at $75^{\circ} \mathrm{C}$. To inactivate the enzymes, EDTA was added to a final concentration of $10 \mathrm{mM}$. To isolate biotin-labelled ligation junctions, $50 \mu \mathrm{l}$ of $10 \mathrm{mg} / \mathrm{ml}$ Dynabeads MyOne Streptavidin C1 (Life Technologies, 65001) were washed with $400 \mu \mathrm{l}$ of $1 \times$ Tween washing buffer (TWB; 5 mM Tris- $\mathrm{HCl}$ pH 7.5, $0.5 \mathrm{mM}$ EDTA, $1 \mathrm{M}$ $\mathrm{NaCl}, 0.05 \%$ Tween 20), collected with a magnet, resuspended in $400 \mu \mathrm{l}$ of $2 \times$ binding buffer ( $10 \mathrm{mM}$ Tris- $\mathrm{HCl} \mathrm{pH} 7.5,1 \mathrm{mM}$ EDTA, $2 \mathrm{M} \mathrm{NaCl}$ ) and added to the sample suspended in $330 \mu \mathrm{l}$ TLE. Biotinylated DNA was bound to the beads by incubating the sample for $15 \mathrm{~min}$ at room temperature with slow rotation. Subsequently, the DNA-bound beads were captured with a magnet, washed twice with $400 \mu \mathrm{l}$ of $1 \times$ binding buffer, washed once in $100 \mu \mathrm{l}$ of $1 \times$ TLE T4 ligase buffer and resuspeded in $41 \mu \mathrm{l}$ of TLE. For polyadenylation, $5 \mu \mathrm{l}$ of $10 \times$ NEBuffer 2.1 , $1 \mu \mathrm{l}$ of $10 \mathrm{mM}$ dATP and $3 \mu \mathrm{l}$ of $5 \mathrm{U} / \mu \mathrm{l}$ of Klenow fragment $\left(3^{\prime} \rightarrow 5^{\prime}\right.$ exo-) (NEB, $\mathrm{M} 0212$ ) and incubated for $30 \mathrm{~min}$ at $37^{\circ} \mathrm{C}$ followed by deactivation for $20 \mathrm{~min}$ at $65^{\circ} \mathrm{C}$. Beads were reclaimed with a magnet, washed once with $400 \mu 11 \times$ Quick ligation buffer (NEB, M2200) and resuspended in $46.5 \mu$ l of $1 \times$ Quick ligation buffer (NEB, M2200). $2.5 \mu \mathrm{l}$ of DNA Quick ligase (NEB, M2200) and $0.5 \mu \mathrm{l}$ of $50 \mu \mathrm{M}$ annealed TruSeq adapters were added and incubated for $1 \mathrm{~h}$ at room temperature. Beads were separated on a magnet, resuspended in $400 \mu \mathrm{l}$ of $1 \times \mathrm{TWB}$ (5 mM Tris- $\mathrm{HCl}, 0.5 \mathrm{M}$ EDTA, $1 \mathrm{M} \mathrm{NaCl}, 0.05 \%$ Tween-20) and washed for $5 \mathrm{~min}$ at room temperature with rotation. Beads were washed on the magnet with $200 \mu \mathrm{l}$ $1 \times$ binding buffer and $200 \mu \mathrm{l}$ of $1 \times$ NEBuffer 2.1 and resuspended in $20 \mu \mathrm{l}$ of $1 \times$ NEBuffer 2.1. The library was amplified in 8 separate reactions of $50 \mu \mathrm{l}$. Per reaction, $1.5 \mu \mathrm{l}$ of $25 \mu \mathrm{M}$ TruSeq PCR primer cocktail (TruSeq PCR primer cocktail_F, TruSeq PCR primer cocktail_R; see Supplementary Table 4), $25 \mu$ of $2 \times$ Kapa HiFi HotStart Ready Mix (Kapa Biosystems, KR0370) and $21.5 \mu \mathrm{l}$ of water were added to $2 \mu \mathrm{l}$ of library bound to the beads. Amplification was performed as follows: $3 \mathrm{~min}$ at $95^{\circ} \mathrm{C}, 5$ cycles of $20 \mathrm{~s}$ at $98^{\circ} \mathrm{C}, 30 \mathrm{~s}$ at $63^{\circ} \mathrm{C}$ and $30 \mathrm{~s}$ at $72{ }^{\circ} \mathrm{C}, 1 \mathrm{cycle}$ of $1 \mathrm{~min}$ at $72^{\circ} \mathrm{C}$, hold at $4{ }^{\circ} \mathrm{C}$. The $\mathrm{PCR}$ reactions were pooled and the beads were removed from the supernatant using a magnet. The library was purified by addition of 1.5 volumes of Agencourt AMPure XP beads (Beckman Coulter), according to the manufacturer's instructions. The sample was eluted off beads using $25 \mu \mathrm{l}$ of $1 \times$ TLE buffer, transferred to a fresh tube and the concentration was determined using Qubit (Qubit dsDNA HS Assay Kit, Thermo Fisher) and qPCR (KAPA SYBR FAST qPCR Master Mix, Kapa Biosystems), according to the manufacturer's instructions. Library size distributions were determined on a $5 \%$ polyacrylamide gel. Paired-end 
76-bp sequencing was carried out using the Illumina NextSeq 500 system with high and mid output NextSeq 500/550 kits according to the manufacturer's instructions. Mapping of $\mathrm{Hi}-\mathrm{C}$ reads and generation of interaction matrices. Reads were trimmed at their ligation junction using the truncator of the HiCUP pipeline ${ }^{37}$ (version 0.5.9 devel), mapped using bwa mem (https://arxiv.org/abs/1303.3997) of the bwa kit version 0.7.12-r1039 and those with a quality score $q>0$ were retained. Reads were mapped to the T. brucei Lister 427 genome version 9 (Tb427v9). For each chromosome, this genome contains the core region of two homologous chromosomes only once, whereas both of the the respective heterozygous subtelomeric regions belonging to the two homologous chromosomes are included. During the mapping of $\mathrm{Hi}-\mathrm{C}$ reads, contigs that displayed alternative variations of an assembled allele were removed (Tb427v9_without_allelic_variants) to keep these loci visible in the $\mathrm{Hi}-\mathrm{C}$ matrices. The primary analysis of $\mathrm{Hi}-\mathrm{C}$ reads was performed with HiC-Pro ${ }^{38}$ (version 2.10.0) to visually inspect reproducibility among replicates. Raw matrices were normalized for differences in ploidy (for example, read counts at diploid regions were multiplied by 0.5 ), balanced by iterative correction using $\mathrm{HiC}$-Pro (default settings) and converted into a homer compatible format ${ }^{39}$ using a custom Python script (see 'Code availability'). To enable comparisons between different Hi-C experiments, each value in the balanced interaction matrix was divided by the respective column sum.

Distance-dependent decay of interaction frequencies. To visualize the distance-dependent decay, interaction frequencies between $\mathrm{Hi}$ - $\mathrm{C}$ bin pairs were grouped on the basis of the linear distance between the pairs, and the distribution of the median interaction frequency across distances was plotted.

Co-localization of genomic loci. To determine whether a region of interest interacted more or less than expected by chance, the median and mean interaction frequencies were calculated for bins overlapping with the regions of interest. In addition, a 'background' interaction frequency was determined by randomly selecting regions of identical size from the same matrix. Significance was determined using Welch's $t$-test.

To identify changes in DNA-DNA interaction frequencies after deletion of histone variants, the ratio of 'feature median interaction frequencies'/'background median interaction frequency' was determined for 100 randomly selected background regions in wild-type, $\Delta H 3 . V, \Delta H 4 . V$ and $\Delta H 3 . V \Delta H 4 . V$ cells. Significance was determined using Welch's $t$-test. Co-localization analyses were performed using balanced interaction matrices (50-kb resolution). Bins with zero values were excluded from the analyses.

4C-like analysis. To visualize interactions between one genomic region (bait) and all other genomic sites, relevant bins were extracted from a $20-\mathrm{kb}$ Hi-C matrix. An average interaction value for every genomic bin was calculated if the bait regions spanned more than one bin.

Hi-C matrix visualization. Matrices were plotted based on the colour palettes provided by seaborn (https://seaborn.pydata.org). To generate differential heat maps, a pseudo-count of 0.000001 was added to each interaction value of the numerator and denominator matrix before division.

SMRT sequencing. Genomic DNA was isolated and precipitated from $3 \times 10^{8}$ cells of the T. brucei 42717.13 P10 isolate ${ }^{40}$ using the Blood \& Cell Culture DNA Midi Kit (Qiagen), according to the manufacturer's instructions. In addition, the DNA was purified in a phenol chloroform extraction using Manual Phase Lock Gel 2-ml (heavy/light) tubes (5Prime) and suspended in $100 \mu \mathrm{l}$ of TE buffer. SMRT library preparation and sequencing was performed at the Icahn School of Medicine at Mount Sinai.

Genomic DNA library preparation and sequencing was performed primarily using the manufacturer's instructions for the P6-C4 sequencing enzyme and chemistries. In short, $\sim 5 \mu \mathrm{g}$ gDNA was quantified and diluted to $150 \mu \mathrm{l}$ using elution buffer (Qiagen) at $33 \mathrm{ng} / \mu \mathrm{l}$ and then sheared to $\sim 20 \mathrm{~kb}$ by centrifugation at 4,500 rpm for $50 \mathrm{~s}$ using a G-tube spin column (Covaris). The sheared DNA was then re-purified using Agencourt AMPure XP beads (Beckman Coulter) at $0.45 \times$. Next, $\sim 1.6$ to $3.2 \mu \mathrm{g}$ of DNA from each batch was taken into DNA damage and end repair. In brief, the DNA fragments were repaired by adding $21.1 \mu \mathrm{l}$ of DNA damage repair solution $(1 \times$ DNA damage repair buffer $(1 \times \mathrm{NAD}+, 1 \mathrm{mM}$ ATP and $0.1 \mathrm{mM} \mathrm{dNTP}$ ) and $1 \times$ DNA damage repair mix) and incubation at $37^{\circ} \mathrm{C}$ for $20 \mathrm{~min}$. DNA ends were repaired by adding $1 \times$ end repair mix to the solution and incubation at $25^{\circ} \mathrm{C}$ for $5 \mathrm{~min}$, followed by an additional $0.45 \times$ Agencourt AMPure XP purification step. Next, $0.75 \mu \mathrm{M}$ of blunt adaptor was added to the DNA and $1 \times$ template preparation buffer $(0.05 \mathrm{mM}$ ATP and $0.75 \mathrm{U} / \mu \mathrm{l} \mathrm{T} 4 \mathrm{DNA}$ ligase) was added to a final volume of $47.5 \mu \mathrm{l}$. This solution was incubated at $25^{\circ} \mathrm{C}$ overnight, followed by incubation at $65^{\circ} \mathrm{C}$ for $10 \mathrm{~min}$ to inactivate the ligase. To remove un-ligated DNA fragments, exonuclease cocktail (1.81 U/ $\mu \mathrm{l}$ Exo III 18 and $0.18 \mathrm{U} / \mu \mathrm{l}$ Exo VII) was added to the library followed by a $60 \mathrm{~min}$ incubation at $37^{\circ} \mathrm{C}$. Two additional $0.45 \times$ Agencourt Ampure XP purification steps were performed to remove $<2,000$-bp molecular weight DNA and organic contaminants.

The size of the library was validated using an Agilent DNA 12000 chip. Before P6-C4-based sequencing, Blue Pippin size selection was applied to remove molecules $<7,000 \mathrm{bp}$. This step was conducted using Sage Science Blue Pippin $0.75 \%$ agarose cassettes to select libraries in the range of 7,000-50,000 bp. Primers were annealed to the size-selected SMRTbell at a ratio of 20:1 with the full-length libraries by denaturation $\left(80^{\circ} \mathrm{C}\right.$ for $\left.2 \mathrm{~min}\right)$ and slow cooling $\left(0.1^{\circ} \mathrm{C} / \mathrm{s}\right.$ to $\left.25^{\circ} \mathrm{C}\right)$. The polymerase-template complex was bound to the $\mathrm{P} 6$ enzyme using a ratio of 10:1 polymerase to SMRTbell at $0.5 \mathrm{nM}$ for $4 \mathrm{~h}$ at $30^{\circ} \mathrm{C}$ and then held at $4{ }^{\circ} \mathrm{C}$ until ready for magnetic-bead loading. The magnetic-bead loading step was conducted at $4{ }^{\circ} \mathrm{C}$ for $60 \mathrm{~min}$. The magnetic-bead-loaded, polymerase-bound SMRTbell libraries were placed onto the RSII machine at a sequencing concentration of $50 \mathrm{pM}$ and configured for a 240-min continuous sequencing run.

Assembly, post-assembly improvements and genome scaffolding. The 642,583 sequencing reads from wild-type gDNA (seven SMRT cells) were assembled into contigs following the RS_HGAP_Assembly.3 workflow from SMRT Analysis v2.3. $0^{41}$ with default parameters. In brief, a sequence seeding dataset with the longest sequencing reads was pulled apart. The remaining reads were mapped onto them to obtain error-corrected reads through a consensus procedure. The error-corrected reads were assembled by traditional overlap layout consensus with a Celera Assembler ${ }^{42}$. Contig sequences were polished with Quiver and were further joined and extended using PBJelly 2 (PBSuite_15.2.20) ${ }^{43}$. Remaining sequence errors were corrected using iCORN $2^{44}$ with previously published and new gDNA Illumina data available under GSM2586510 (https://www.ncbi.nlm. nih.gov/geo/query/acc.cgi?acc=GSM2586510) and ERS1503958 (http://www.ebi. ac.uk/ena/data/view/ERS1503958), respectively.

For genome scaffolding, all Hi-C reads were combined, mapped onto the error-corrected HGAPv3 contigs and a balanced $10-\mathrm{kb}$ bin heat map matrix of DNA-DNA interactions was generated as previously described. For the scaffolding, only a subset of the matrix that contained contigs larger than $50 \mathrm{~kb}$ was considered (Extended Data Fig. 1).

Then, contigs that did not exhibit a gradual distance-dependent decay in DNADNA interactions (which suggests that they may have been incorrectly assembled) were broken at the site of the putative mis-assembly. Available pipelines for the scaffolding of contigs based on DNA-DNA interaction frequencies are not designed for genomes that contain large regions of heterozygosity ${ }^{45,46}$; therefore, contigs were manually rearranged and/or inverted so that long-distance DNA interactionsvisible as signal away from the diagonal-were reduced. The process of $\mathrm{Hi}-\mathrm{C}$ read-mapping, heat map generation and contig repositioning or inversion was repeated until no contigs could be identified, the repositioning of which would have further minimized the signal away from the diagonal (Extended Data Fig. 1). To validate our scaffolding approach and to further improve the genome, we ran PBJelly 2, which enabled us to join together several of the contigs that we had placed next to each other and to reduce the number of contigs from 139 to 91 . In addition, we compared the obtained Lister 427 scaffold to that of the previously assembled TREU 927 genome and found that the core regions exhibited a high degree of similarity and synteny (Extended Data Fig. 2), which validates our scaffolding approach. The subtelomeric regions are known to be different between the genomes. Finally, we observed that 27 out of the 33 core-subtelomere boundaries in the assembly are spanned by PacBio reads and/or contigs, which supports their linear proximity (see Supplementary Information).

For a comparison of different assembly strategies and an assessment of the genome quality, see Supplementary Information and Supplementary Table 3. The genome-built $\mathrm{Tb} 427 \mathrm{v} 9$, which was used for all analyses performed in this study, is available in the European Nucleotide Archive with the ENA study accession number PRJEB18945 (http://www.ebi.ac.uk/ena/data/view/PRJEB18945).

Genome annotation. Annotations from the T. brucei TREU 927 genome were transferred using Companion (https://companion.sanger.ac.uk; accessed 9 January $2017)^{47}$. In addition, VSG genes were annotated based on similarity ( $>90 \%$ coverage and $>95 \%$ identity to a VSG gene) of the available VSGnome dataset from the Lister 427 strain $^{10}$ with NCBI-BLAST+ (version 2.2.31+). Overlapping VSG-gene entries were merged and named after the entry with the best bit score. For the identification of putative novel MESs, the assignment of BESs to chromosome ends and the identification of centromeres, see Supplementary Information.

Chromatin immunoprecipitation with sequencing. Assays were performed as previously described ${ }^{48}$ with minor alterations. For the immunoprecipitation of cell lines with Ty1-tagged proteins (Ty1-H3.V/DH3.V, Ty1-H4.V $\Delta H 4 . V / \Delta H 4 . V$ and Ty1-SCC1/ $\triangle S C C 1$ cells), $50 \mu$ l of Dynabeads protein $\mathrm{G}$ (ThermoFisher) was separated on a magnet, the supernatant was removed and the beads were resuspended in $200 \mu \mathrm{l}$ of PBS-Tween $(0.02 \%)$ containing $10 \mu \mathrm{g}$ of BB2 antibody ${ }^{49}$, and then incubated with slow rotation at $4{ }^{\circ} \mathrm{C}$ overnight. Antibody-coupled protein $\mathrm{G}$ beads were separated on a magnetic rack and washed three times with PBS-Tween $(0.02 \%)$. Five hundred microlitres of ChIP sample was added, and incubated at $4{ }^{\circ} \mathrm{C}$ overnight with slow rotation. For ChIP assessment of the distribution of histone variants, the DNA was fragmented using micrococcal nuclease; for ChIP assessment of the distribution of SCC1, the DNA was sheared using a Covaris S220 instrument before target protein binding. Sequencing reads were mapped using 
bwa mem and coverage plots were generated using COVERnant (v.0.3.2) (https:// github.com/konrad/COVERnant) ${ }^{13}$.

ATAC-seq. To ensure reproducibility of the assays independent of cell-number variations, all assays were performed with $1 \times 10^{6}$ and $2 \times 10^{6}$ cells. To this end $3 \times 10^{7}$ cells were collected and washed in $30 \mathrm{ml}$ of cold $1 \times \mathrm{TDB}$. The pellet was resuspended in $300 \mu \mathrm{l}$ permeabilization buffer with protease inhibitors, $3 \mu \mathrm{l}$ of $4 \mathrm{mM}$ digitonin was added and incubated for $5 \mathrm{~min}$ at room temperature. The cells were pelleted, resuspended in $600 \mu \mathrm{l}$ isotonic buffer with protease inhibitors and split in two samples, containing $1 \times 10^{7}$ and $2 \times 10^{7}$ cells, respectively. The transposition reaction was performed by adding $50 \mu \mathrm{l}$ of transposition mix to the pellet $(25 \mu \mathrm{lDD}(2 \times$ reaction buffer from Nextera kit), $25 \mu \mathrm{l}$ TDE1 (Tn 5 transposase from Nextera kit), $22.5 \mu \mathrm{l}$ nuclease-free water) and incubation for $30 \mathrm{~min}$ at $37^{\circ} \mathrm{C}$. For the gDNA control, $200 \mathrm{ng}$ of gDNA was treated in the same manner. The DNA samples were purified using Qiagen MinElute PCR Purification Kit and eluted in $10 \mu \mathrm{l} \mathrm{EB}(10 \mathrm{mM}$ Tris- $\mathrm{HCl}, \mathrm{pH} 8)$. The transposed DNA fragments were amplified using the NEBNext High-Fidelity $2 \times$ PCR Master Mix (M0541) supplied with $2.5 \mu \mathrm{l}$ of $25 \mu \mathrm{M}$ barcoded primers and amplification for 13 cycles. The libraries were purified using AMPure XP beads (Beckman Coulter) according to the manufacturer's instructions. The library fragment sizes between 150 and 1,000 bp were purified from a $6 \%$ polyacrylamide gel. Paired-end 76-bp sequencing was carried out using the Illumina NextSeq 500 system with a high-output NextSeq $500 / 550 \mathrm{kit}$, according to the manufacturer's instructions. Sequencing reads were mapped using bwa mem and coverage plots were generated using COVERnant (v.0.3.2) (https://github.com/konrad/COVERnant) ${ }^{13}$

scRNA-seq. T. brucei wild-type and $\Delta H 3 . V \Delta H 4 . V$ cells were sorted ( 0 cell, 1 cell or 50 cells) using a FACSAria III (BD Biosciences; precision: single-cell; nozzle: $100 \mu \mathrm{m})$. A forward-scatter area versus side-scatter area plot was used to gate and sort the cells. T. brucei cells were sorted in 48-well plates (Brand) filled with $2.6 \mu \mathrm{l}$ of $1 \times$ lysis buffer (Takara) supplemented with $0.01 \mu \mathrm{l}$ of RNase inhibitor

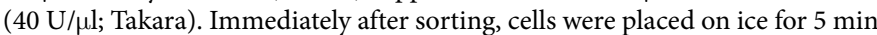
and stored at $-80^{\circ} \mathrm{C}$.

Lysates from 50 trypanosomes and single trypanosomes were supplemented with $0.2 \mu \mathrm{l}$ of a $1: 2 \times 10^{6}$ (scRNA-seq I) or a 1:20 $\times 10^{6}$ (scRNA-seq II) dilution of ERCC Spike-In Control Mix 1 (Thermo Fisher, 4456740). Libraries were prepared using SMART-Seq v.4 Ultra Low Input RNA Kit (Takara) using a quarter of the reagent volumes recommended by the manufacturer. PCR amplification was performed using 26 cycles (scRNA-seq I) or 22 cycles (scRNA-seq II), according to the supplier's recommendations. cDNA was purified using Agencourt AMPure XP beads (Beckman Coulter) and recovered in $15 \mu$ l of elution buffer (Takara). Libraries were quantified using the Qubit 3 Fluorometer with dsDNA Hs Assay kit (Life Technologies) and the quality of the libraries was assessed using a 2100 Bioanalyzer with High Sensitivity DNA kit (Agilent) (Extended Data Fig. 7). Similar to previously published approaches ${ }^{50}, 0.5 \mathrm{ng}$ of cDNA was subjected to a tagmentation-based protocol (Nextera XT, Illumina) using a quarter of the recommended volumes, $10 \mathrm{~min}$ for tagmentation at $55^{\circ} \mathrm{C}$ and $1 \mathrm{~min}$ extension time during PCR amplification. Libraries were pooled (96 libraries for NextSeq) and sequencing was performed in paired-end mode for $2 \times 75$ cycles using Illumina's NextSeq 500. Details of the sequencing results are listed in Supplementary Table 2.

Analysis of scRNA-seq. The reads were mapped to the combination of the Tb427v9 genome with the ERCC spike-in sequences, using bwa mem, version 0.7.16. The mapped data were processed using samtools ${ }^{51}$ (version 1.8) and MarkDuplicates tool (2.18.3-SNAPSHOT) from Picard (http://broadinstitute.github.io/picard/), and the read counts-to-features were done with bedtools ${ }^{52}$ (version 2.26.0). To assess the quality of the data, for each scRNA-seq experiment the read counts to the following groups were determined: reads mapping to 'rRNA genes', reads mapping to 'protein-coding genes' (CDS plus $89 \mathrm{bp}$ for the $5^{\prime}$ UTR and $400 \mathrm{bp}$ for the $\left.3^{\prime} \mathrm{UTR}\right)^{53}$, reads mapping to 'other regions of the genome' and 'unmapped reads'. Reads that overlapped 'rRNA genes' and 'protein-coding genes' features at the same time were excluded from both groups and counted as reads mapping to 'other regions of the genome'.

To assess the library complexity of the scRNA-seq datasets, the number of genes with $\geq 10$ read counts was determined for each cell. The counts per feature group, as well as the number of genes captured in each scRNA-seq experiment, are available in Supplementary Table 2. Only those scRNA-seq datasets with more than 500 genes with $\geq 10$ read counts were considered for the quantification of VSG gene expression.

Many VSG genes share a high degree of homology with each other. Therefore, to determine the expression levels for each of the 2,846 VSG genes annotated in $\mathrm{Tb} 427 \mathrm{v} 9$, the number of uniquely mapping sequence reads obtained for each VSG gene was normalized to account for differences in uniqueness. The uniqueness of the VSG genes was determined by alignment of an in silico-generated dataset that matched the scRNA-seq datasets in read size and fragment-length distribution. For each cell, the transcript level of an individual VSG gene is shown as a percentage of the total VSG gene transcript level in that cell. Raw and normalized read counts are available in Supplementary Table 2, and a diagram explaining the VSG count normalization procedure is shown in Extended Data Fig. 7.

Total RNA-seq. Triplicates of T. brucei wild-type, $\Delta H 3 . V, \Delta H 4 . V$ and $\Delta H 3$. $V \Delta H 4 . V$ cells were grown to a density of $\sim 10^{6}$ cells $/ \mathrm{ml}$. Cell concentration was determined nine times for each replicate using a Coulter Cell Counter (Beckman Coulter) and $4.5 \times 10^{7}$ cells were collected from each culture. Cells were washed with $1 \times$ TDB, resuspended in $350 \mu$ l of buffer RA1 (Macherey-Nagel, NucleoSpin RNA) and $38 \mu \mathrm{l}$ of $0.1 \mathrm{M}$ DTT and $1 \mu \mathrm{l}$ of a 1:10 dilution of ERCC Spike-In Control Mix (Thermo Fisher, 4456740) were added. Total RNA was purified from the lysate according to the NucleoSpin RNA kit protocol, and eluted in $30 \mu$ of nuclease-free water. To deplete rRNA, $3.5 \mu \mathrm{g}$ of total RNA was mixed with $2.6 \mu \mathrm{l}$ of $5 \times$ hybridization buffer (500 mM Tris- $\mathrm{HCl} \mathrm{pH} \mathrm{7.4,1} \mathrm{M} \mathrm{NaCl),} 0.459$ pmol of 131 50-bp anti-rRNA oligonucleotides (covering $18 \mathrm{~S}, \mathrm{M1}, \mathrm{M} 2, \mathrm{M3}, \mathrm{M4}, \mathrm{M5}, \mathrm{M6}, 28 \mathrm{~S}$ alpha and $28 S$ beta rRNAs, a kind gift of C. Clayton; for the full list, see Supplementary Table 4) and $2.2 \mu \mathrm{l}$ of water, denatured at $95^{\circ} \mathrm{C}$ for $2 \mathrm{~min}$ and slowly $\left(0.1^{\circ} \mathrm{C}\right.$ per s) cooled to $37^{\circ} \mathrm{C}$. One microlitre of prewarmed $10 \times$ RNaseH digestion buffer $(200 \mathrm{mM}$ Tris-HCl, pH 7.4, $500 \mathrm{mM} \mathrm{KCl}, 40 \mathrm{mM} \mathrm{MgCl}_{2}, 10 \mathrm{mM} \mathrm{DTT}$ ) and $10 \mathrm{U}$ of RNaseH (ThermoFisher, AM2292) were added and the volume was adjusted to $16 \mu \mathrm{l}$ with nuclease-free water. The mixture was incubated at $37^{\circ} \mathrm{C}$ for $20 \mathrm{~min}$. Residual oligonucleotides were subsequently digested by addition of $2 \mathrm{U}$ of Turbo DNaseI and $5 \mu \mathrm{l}$ of $10 \times$ Turbo DNase reaction buffer (AM2238, Thermo Fisher) in a total reaction volume of $50 \mu \mathrm{l}$ and incubation at $37^{\circ} \mathrm{C}$ for $20 \mathrm{~min}$. The DNase was inactivated by addition of EDTA ( $15 \mathrm{mM}$ final concentration) and heating at $75^{\circ} \mathrm{C}$ for $10 \mathrm{~min}$. The RNA was purified using RNAeasy Minelute columns (Qiagen), according to the manufacturer's instructions, and eluted in $14 \mu \mathrm{l}$ of RNase-free water. Double-stranded cDNA was synthesized from $100 \mathrm{ng}$ of rRNA-depleted RNA using the NEBNext Ultra RNA Library Prep Kit for Illumina. The double-stranded cDNA was purified using Agencourt AMPure XP beads (Beckman Coulter) and eluted in 60 or $30 \mu \mathrm{l}$ of $0.1 \times$ TE Buffer, respectively. Libraries were prepared and sequenced as previously described ${ }^{48}$.

Analysis of RNA-seq data. After adaptor clipping and quality trimming using cutadapt $^{54}$ (version 1.10), the RNA-seq reads were mapped against the T. brucei genome using bwa mem of the bwa kit version 0.7.16. Only reads with a quality score $q>0$ were retained. Feature quantification was performed with bedtools multicov subcommand. Differential gene expression analysis was then conducted with DESeq2 (v.1.20.0) ${ }^{55}$. Features with an adjusted $P$ value (calculated based on Wald test and adjusted for multiple testing using the procedure of Benjamini and Hochberg ${ }^{56}$ ) below 0.1 were considered as differentially expressed. Supplementary Table 1 contains the raw counts for each gene in each individual RNA-seq replicate, as well as the fold change (in $\log _{2}$ scale) and $P$ value adjusted for each sample versus wild type.

Fluorescence in situ hybridization. For each FISH assay, $1 \times 10^{7}$ bloodstreamform trypanosomes grown to a density of up to $1 \times 10^{6} \mathrm{cells} / \mathrm{ml}$ were collected and the cell pellet was washed once with $1 \times$ TDB and fixed for $15 \mathrm{~min}$ in $1 \times$ TDB containing $4 \%$ formaldehyde. Cells were washed with $1 \times$ TDB once and resuspended in $50 \mu \mathrm{l}$ of $1 \times$ TDB. Gene frames were placed onto microscopy slides to cover an aminopropyltriethoxysilane-coated coverslip. Cells were pipetted onto the framed coverslip and allowed to settle for $5 \mathrm{~min}$ by gravity. The sample was washed twice for 2 min with $90 \mu \mathrm{l}$ of $1 \times \mathrm{TDB}$, incubated with quenching solution $\left(1 \times \mathrm{TDB}\right.$ containing $\left.1 \mathrm{mg} / \mathrm{ml} \mathrm{NaBH}_{4}\right)$ for $10 \mathrm{~min}$, washed twice with $1 \times \mathrm{TDB}$, permeabilized with $70 \mu \mathrm{l}$ of $1 \times \mathrm{TDB}$ containing $0.1 \% \mathrm{NP}-40$ for $5 \mathrm{~min}$ and washed twice with $1 \times$ TDB. Next, cells were treated with $1 \times$ PBS containing $1 \mathrm{mg} / \mathrm{ml}$ RNaseA for $30 \mathrm{~min}$, washed twice with $1 \times$ TDB and incubated with $50 \mu \mathrm{l}$ of a 1:1 dilution of hybridization buffer with $2 \times \mathrm{SSC}$ for $30 \mathrm{~min}$ at room temperature. The labelled probe (see Supplementary Table 4) was diluted to a final concentration of $400 \mathrm{nM}$ in $25 \mu$ lof hybridization buffer (50 (v/v) formamide, $10 \%(\mathrm{w} / \mathrm{v})$ dextran sulfate, $2 \times$ SSPE, $250 \mu \mathrm{g} / \mathrm{ml}$ herring sperm DNA). The hybridization buffer was removed from the sample, $25 \mu \mathrm{l}$ of hybridization solution containing the probe was added and the frame was closed with a plastic lid. The sample was incubated using a thermal block at $90^{\circ} \mathrm{C}$ for $5 \mathrm{~min}$ and at $37^{\circ} \mathrm{C}$ overnight. Next, the samples were washed for $30 \mathrm{~min}$ in $30 \mathrm{ml}$ of $50 \%(\mathrm{v} / \mathrm{v})$ deionized formamide and $2 \times S S C$ at $37^{\circ} \mathrm{C}$, for $10 \mathrm{~min}$ in $30 \mathrm{ml}$ of $1 \times \mathrm{SSC}$ at $50^{\circ} \mathrm{C}$, for $10 \mathrm{~min}$ in $30 \mathrm{ml}$ of $2 \times \mathrm{SSC}$ at $50^{\circ} \mathrm{C}$ and for $10 \mathrm{~min}$ in $30 \mathrm{ml}$ of $4 \times \mathrm{SSC}$ at room temperature. Subsequently, cells were blocked in P1 buffer (100 mM maleic acid, $150 \mathrm{mM}$ sodium chloride, $\mathrm{pH} 7.5$, $4 \%$ BSA, $1 \%$ milk) for $1 \mathrm{~h}$, incubated with primary antibody (sheep anti-digoxigenin antigen-binding fragment, Roche, diluted 1:2,000 in P1) for 45-60 min and washed with $0.5 \%$ Tween-20 and PBS 4 times for 4 min each. Cells were then incubated with the secondary antibody (Alexa Fluor 488 conjugated donkey anti-sheep IgG $(\mathrm{H}+\mathrm{L})$, Life Technologies, A11015, diluted 1:2,000 in P1) for 30-60 min. For further signal amplification, the samples were washed with PBS containing $0.5 \%$ Tween-20 4 times for $4 \mathrm{~min}$ and incubated with a rabbit anti-donkey $\operatorname{IgG}(\mathrm{H}+\mathrm{L})$ DyLight 488 (Invitrogen) (diluted 1:2,000 in P1) for 30-60 min. The samples were washed twice for $10 \mathrm{~min}$ in $30 \mathrm{ml}$ of PBS containing $0.5 \%$ Tween-20 and another $10 \mathrm{~min}$ in $30 \mathrm{ml}$ of PBS, each time in a falcon tube on a shaker. Samples were 
mounted with $36 \mu \mathrm{l}$ of Vectashield Mounting Medium with DAPI (Biozol) on a microscopy slide and were sealed with nail polish.

Immunofluorescence. Immunofluorescence was performed as previously described ${ }^{57}$, with minor alterations. Ten million cells per $\mathrm{ml}$ (wild type, $\Delta H 3 . V$, $\Delta H 4 . V$ and $\Delta H 3 . V \Delta H 4 . V$ ) were suspended in HMI-11 containing $2 \%$ formaldehyde, incubated for $5 \mathrm{~min}$ at room temperature and washed with $1 \times \mathrm{TDB}$. $\alpha$-Tubulin was stained using the mouse monoclonal antibody Tat $1^{58}(1: 200)$ and a secondary Alexa Fluor 594-conjugated chicken anti-mouse IgG (1:350, Invitrogen). VSG-2 was stained using CRD-depleted rabbit anti-VSG-2 ${ }^{40}(1: 1,000)$ and a secondary Alexa Fluor 488-conjugated donkey anti-rabbit IgG (1:350, Invitrogen).

Fluorescence microscopy and image analysis. For imaging, a wide-field fluorescence Leica DMI6000 microscope with a mercury metal halide lamp and a HCX PL APO CS $100 \times / 1.47$ OIL objective was used. Images were captured with a Leica DFC 360 FX camera. Stacks with 32 slices and $6.3232 \mu \mathrm{m}$ in height $(0.0645$ $\times 0.0644 \times 0.1976 \mu \mathrm{m}^{3}$ voxel size) were captured using identic exposure times for all conditions.

Quantification of 'large' telomere clusters was carried out using Imaris 8 software (Oxford Instruments). After segmenting individual nuclei in the DAPI channel, surfaces were rendered for the telomere FISH signal, while setting the quality filter $>1,000$. All FISH signals that generated surfaces with a volume $>0.3 \mu \mathrm{m}^{3}$ were classified as large telomere clusters, and scored.

FACS flow cytometry. VSG expression on the cell surface was quantified according to a previously published protocol ${ }^{40}$. In brief, $1 \times 10^{6}$ cells were centrifuged in a chilled microcentrifuge at $1,500 \mathrm{~g}$ for $4 \mathrm{~min}$ at $4{ }^{\circ} \mathrm{C}$. Cells were resuspended in $100 \mu \mathrm{l}$ of ice cold HMI-11 and a VSG-specific antibody (anti-VSG-2 or anti-VSG-13) ${ }^{40}$ was added. After $60 \mathrm{~min}$ of incubation at $4{ }^{\circ} \mathrm{C}$ with gentle shaking, cells were washed three times in $500 \mu \mathrm{l}$ of ice cold HMI-11, resuspended in $100 \mu \mathrm{l}$ of cold HMI-11 and incubated with an Alexa Fluor 488-conjugated secondary antibody for $20 \mathrm{~min}$. The cells were washed twice with $500 \mu \mathrm{l}$ of $1 \times$ TDB and finally resuspended in $400 \mu \mathrm{l}$ of $1 \times$ TDB before analysis with a FACSort flow cytometer (Becton Dickinson Biosciences).

To determine the cell-cycle profiles, $5 \times 10^{6}$ cells were collected $(10 \mathrm{~min}, 1,300 \mathrm{~g}$, $4^{\circ} \mathrm{C}$ ) and washed once with ice-cold $1 \times \mathrm{TDB}$. The cells were resuspended in $1 \mathrm{ml}$ ice-cold PBS and $2 \mathrm{mM}$ EDTA and fixed by adding $2.5 \mathrm{ml}$ ice-cold methanol. After a 1-h incubation, cells were washed with $1 \mathrm{ml}$ PBS and EDTA at room temperature and resuspend in $1 \mathrm{ml} \mathrm{PBS}$ and EDTA. One microlitre RNaseA $(10 \mu \mathrm{g} / \mu \mathrm{l})$ and $10 \mu \mathrm{l}$ propidium iodide $(1 \mu \mathrm{g} / \mu \mathrm{l})$ were added. The stained cells were analysed with a FACSCalibur (Becton Dickinson) after a 30-min incubation at $37^{\circ} \mathrm{C}$.

Mapping the site of recombination. A library from $\Delta H 3 . V \Delta H 4 . V$ gDNA was prepared as described for wild-type gDNA and sequenced using the PacBio Sequel system by diffusion at $5 \mathrm{pM}$, with v2.1 chemistry and a 10 -h movie. Reads $>10 \mathrm{~Kb}$ were extracted, split into $\sim 2,500$-bp chunks and mapped independently to the genome. Reads that contained chunks that mapped to BES1 and BES15 were kept and mapped again to BES1 and BES15. Based on the observed mapping pattern, a BES1-BES15 hybrid was constructed.

Code availability. Workflows and custom-made Unix Shell, Python and R scripts have been deposited at Zenodo (https://doi.org/10.5281/zenodo.823671). Documentation to reproduce the data analysis is provided.

Reporting summary. Further information on research design is available in the Nature Research Reporting Summary linked to this paper.

\section{Data availability}

The RNA-seq, scRNA-seq, ChIP-seq, ATAC-seq and Hi-C sequencing data have been deposited in the Gene Expression Omnibus ${ }^{59}$ and are accessible through GEO Series accession number GSE100896. The raw SMRT sequencing reads and the genome assembly have been deposited in the European Nucleotide Archive and are accessible through ENA study accession number PRJEB18945. All other data are available from the corresponding author upon reasonable request.

31. Hirumi, H. \& Hirumi, K. Continuous cultivation of Trypanosoma brucei blood stream forms in a medium containing a low concentration of serum protein without feeder cell layers. J. Parasitol. 75, 985-989 (1989).
32. Lowell, J. E. \& Cross, G. A. A variant histone $\mathrm{H} 3$ is enriched at telomeres in Trypanosoma brucei. J. Cell Sci. 117, 5937-5947 (2004).

33. Scahill, M. D., Pastar, I. \& Cross, G. A. M. CRE recombinase-based positivenegative selection systems for genetic manipulation in Trypanosoma brucei. Mol. Biochem. Parasitol. 157, 73-82 (2008).

34. Wirtz, E., Leal, S., Ochatt, C. \& Cross, G. A. M. A tightly regulated inducible expression system for conditional gene knock-outs and dominant-negative genetics in Trypanosoma brucei. Mol. Biochem. Parasitol. 99, 89-101 (1999).

35. Rao, S. S. et al. A 3D map of the human genome at kilobase resolution reveals principles of chromatin looping. Cell 159, 1665-1680 (2014).

36. Belaghzal, H., Dekker, J. \& Gibcus, J. H. Hi-C 2.0: An optimized Hi-C procedure for high-resolution genome-wide mapping of chromosome conformation. Methods 123, 56-65 (2017).

37. Wingett, S. et al. HiCUP: pipeline for mapping and processing Hi-C data. F1000Res. 4, 1310 (2015).

38. Servant, N. et al. HiC-Pro: an optimized and flexible pipeline for $\mathrm{Hi}-\mathrm{C}$ data processing. Genome Biol. 16, 259 (2015).

39. Heinz, S. et al. Simple combinations of lineage-determining transcription factors prime cis-regulatory elements required for macrophage and B cell identities. Mol. Cell 38, 576-589 (2010).

40. Figueiredo, L. M., Janzen, C. J. \& Cross, G. A. M. A histone methyltransferase modulates antigenic variation in African trypanosomes. PLoS Biol. 6, e161 (2008).

41. Chin, C. S. et al. Nonhybrid, finished microbial genome assemblies from long-read SMRT sequencing data. Nat. Methods 10, 563-569 (2013)

42. Myers, E. W. et al. A whole-genome assembly of Drosophila. Science 287, 2196-2204 (2000).

43. English, A. C. et al. Mind the gap: upgrading genomes with Pacific Biosciences RS long-read sequencing technology. PLoS ONE 7, e47768 (2012).

44. Otto, T. D., Sanders, M., Berriman, M. \& Newbold, C. Iterative Correction of Reference Nucleotides (iCORN) using second generation sequencing technology. Bioinformatics 26, 1704-1707 (2010).

45. Kaplan, N. \& Dekker, J. High-throughput genome scaffolding from in vivo DNA interaction frequency. Nat. Biotechnol. 31, 1143-1147 (2013).

46. Burton, J. N. et al. Chromosome-scale scaffolding of de novo genome assemblies based on chromatin interactions. Nat. Biotechnol. 31, 1119-1125 (2013).

47. Steinbiss, S. et al. Companion: a web server for annotation and analysis of parasite genomes. Nucleic Acids Res. 44, W29-W34 (2016).

48. Wedel, C. \& Siegel, T. N. Genome-wide analysis of chromatin structures in Trypanosoma brucei using high-resolution MNase-ChIP-seq. Exp. Parasitol. 180, 2-12 (2017).

49. Bastin, P., Bagherzadeh, Z., Matthews, K. R. \& Gull, K. A novel epitope tag system to study protein targeting and organelle biogenesis in Trypanosoma brucei. Mol. Biochem. Parasitol. 77, 235-239 (1996).

50. Patel, A. P. et al. Single-cell RNA-seq highlights intratumoral heterogeneity in primary glioblastoma. Science 344, 1396-1401 (2014).

51. Li, H. et al. The Sequence Alignment/Map format and SAMtools. Bioinformatics 25, 2078-2079 (2009)

52. Quinlan, A. R. \& Hall, I. M. BEDTools: a flexible suite of utilities for comparing genomic features. Bioinformatics 26, 841-842 (2010).

53. Siegel, T. N., Hekstra, D. R., Wang, X., Dewell, S. \& Cross, G. A. M. Genome-wide analysis of mRNA abundance in two life-cycle stages of Trypanosoma brucei and identification of splicing and polyadenylation sites. Nucleic Acids Res. 38, 4946-4957 (2010).

54. Martin, M. Cutadapt removes adapter sequences from high-throughput sequencing reads. EMBnet J. 17, 10-12 (2011).

55. Love, M. I., Huber, W. \& Anders, S. Moderated estimation of fold change and dispersion for RNA-seq data with DESeq2. Genome Biol. 15, 550 (2014).

56. Benjamini, Y. \& Hochberg, Y. Controlling the false discovery rate: a practical and powerful approach to multiple testing. J. R. Stat. Soc. Ser. B 57, 289-300 (1995).

57. Siegel, T. N. et al. Acetylation of histone H4K4 is cell cycle regulated and mediated by HAT3 in Trypanosoma brucei. Mol. Microbiol. 67, 762-771 (2008).

58. Woods, A. et al. Definition of individual components within the cytoskeleton of Trypanosoma brucei by a library of monoclonal antibodies. J. Cell Sci. 93 491-500 (1989)

59. Edgar, R., Domrachev, M. \& Lash, A. E. Gene Expression Omnibus: NCBI gene expression and hybridization array data repository. Nucleic Acids Res. 30, 207-210 (2002).

60. Carver, T. J. et al. ACT: the Artemis Comparison Tool. Bioinformatics $\mathbf{2 1}$ 3422-3423 (2005). 
a

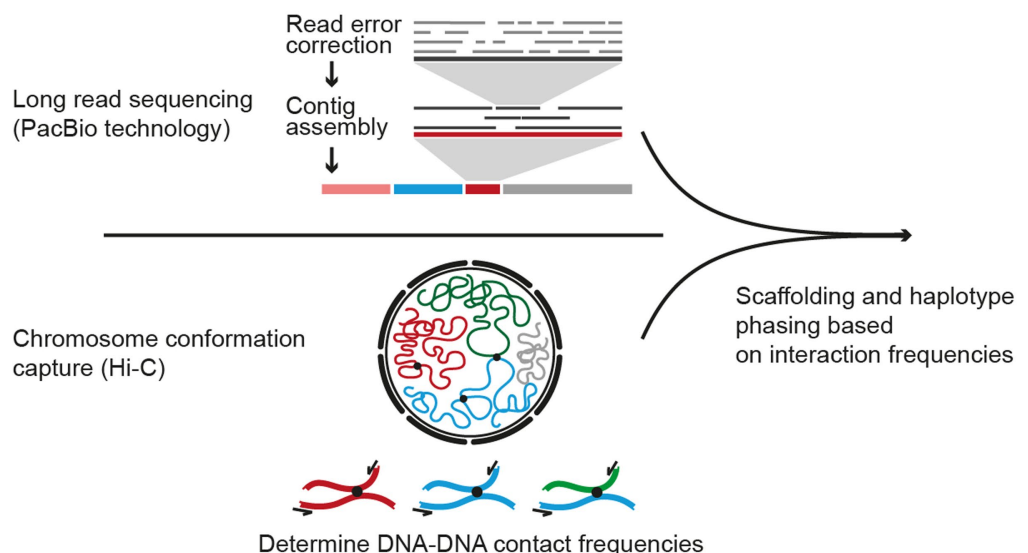

Partially phased genome

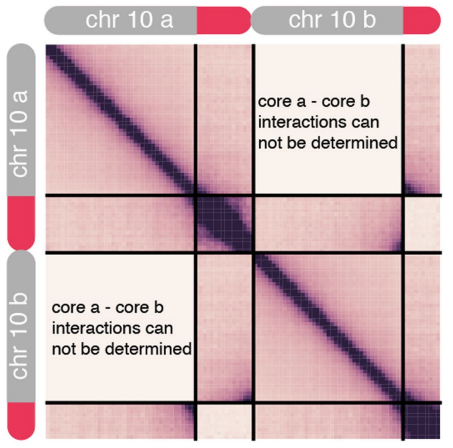

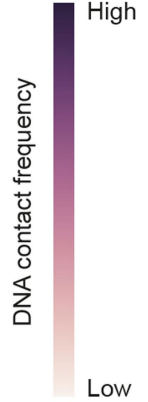

b

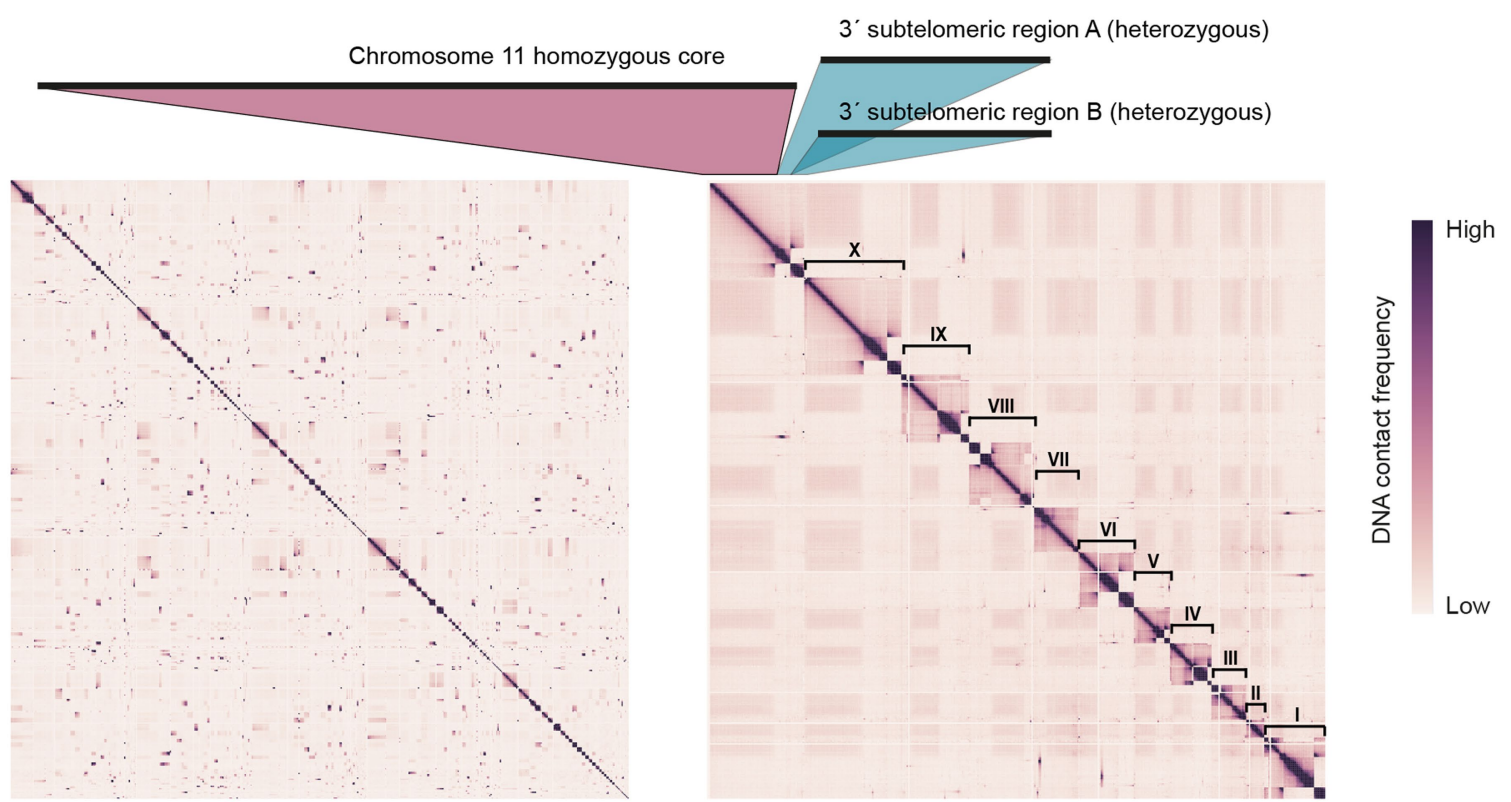

Before scaffolding of contigs

After scaffolding of contigs based on DNA-DNA contact frequencies

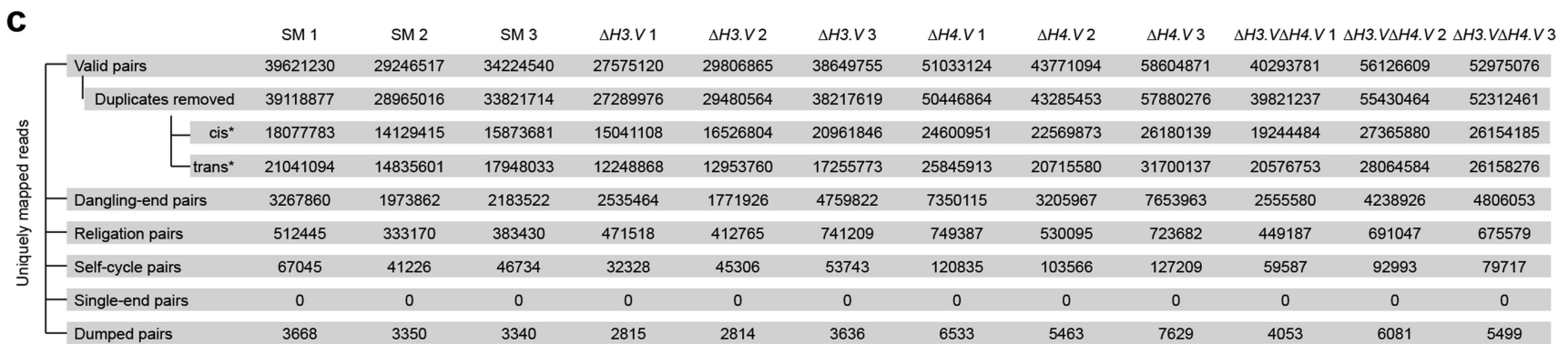

Extended Data Fig. 1 | Assembly of the T. brucei Lister 427 genome. a, Outline of the genome-assembly strategy: gDNA of T. brucei Lister 427 was sequenced using SMRT sequencing technology and P6-C4 sequence chemistry. The $10 \%$ longest reads were error-corrected using the remaining SMRT reads and assembled into contigs using the HGAPv3 algorithm ${ }^{41}$. Information on spatial contacts between contigs, obtained from $\mathrm{Hi}-\mathrm{C}$ analyses, was used to position and orient the contigs into scaffolds. $\mathbf{b}$, To scaffold and orient the contigs, Hi-C reads were mapped to 1,232 contigs to generate a heat map of DNA-DNA interactions (left). Scaffolding was performed by placing contigs such that the interaction signal located away from the diagonal could not be further reduced (right). Heterozygous subtelomeric regions displayed strong interactions with the chromosomal core region but not with other subtelomeric regions, which indicates that they belong to independent homologous chromosomes.

Note that for the left arm of chromosome 7, the heterozygous subtelomeric regions of the two homologous chromosomes could not be assembled separately. c, Statistics of $\mathrm{Hi}-\mathrm{C}$ data analysis based on reads mapped to a joined genome version (haploid A-forks joined to the core). This implies an underestimation of $c i s$, and overestimation of trans interactions (marked with asterisks), as the B-forks remain un-joined. 
a

Synteny between homologous chromosomes (Lister 427)

$\mp$
$\frac{5}{0}$

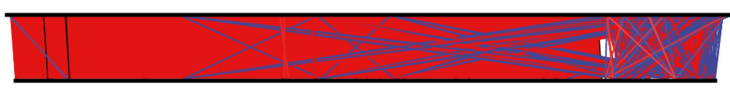

음
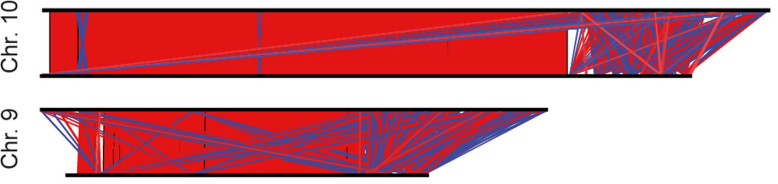

$\infty$
$\frac{1}{0}$
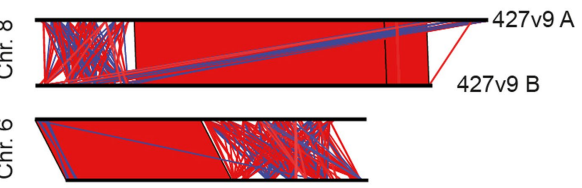

它
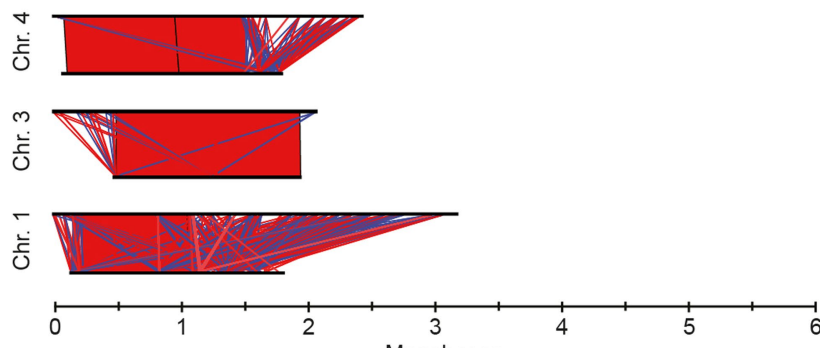

Extended Data Fig. 2 | Synteny between homologous chromosomes and between different isolates. a, Pairwise comparison of corresponding homologous chromosomes using the Artemis Comparison Tool (ACT) of the Wellcome Trust Sanger Institute ${ }^{60}$. Pairs of regions that share a high degree of similarity (BLAST score $\geq 5,000$ ) are connected by boxes in red, or in blue if they are inverted. Chromosome 7 is not shown because the subtelomeric regions of the two homologous chromosomes are very b

Synteny between Lister 427 and TREU 927
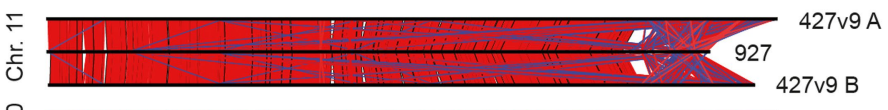

西

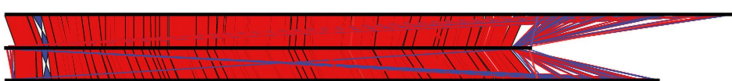
$427 v 9 \mathrm{~B}$
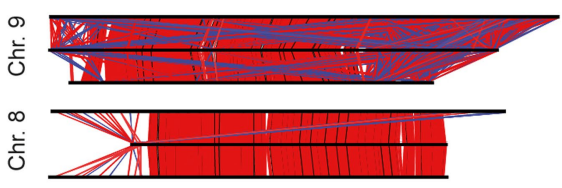

זे

这
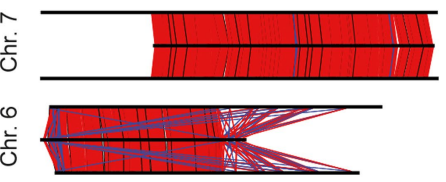

ำ

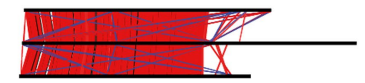

它
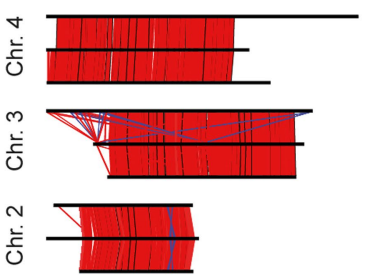

亡

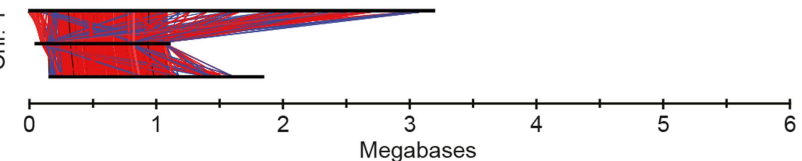

similar and could not be resolved during the assembly. Chromosome 2 is not shown as only one of the two homologous chromosomes contains an extended subtelomeric region. $\mathbf{b}$, Pairwise comparison of the eleven megabase-chromosomes of the TREU 927 isolate (middle black bar) and the corresponding two homologous chromosomes of the Lister 427 isolate (top and bottom black bars) using $\mathrm{ACT}^{60}$. Regions that reached a BLAST score of at least 5,000 are drawn in red, or in blue if they are inverted. 
a
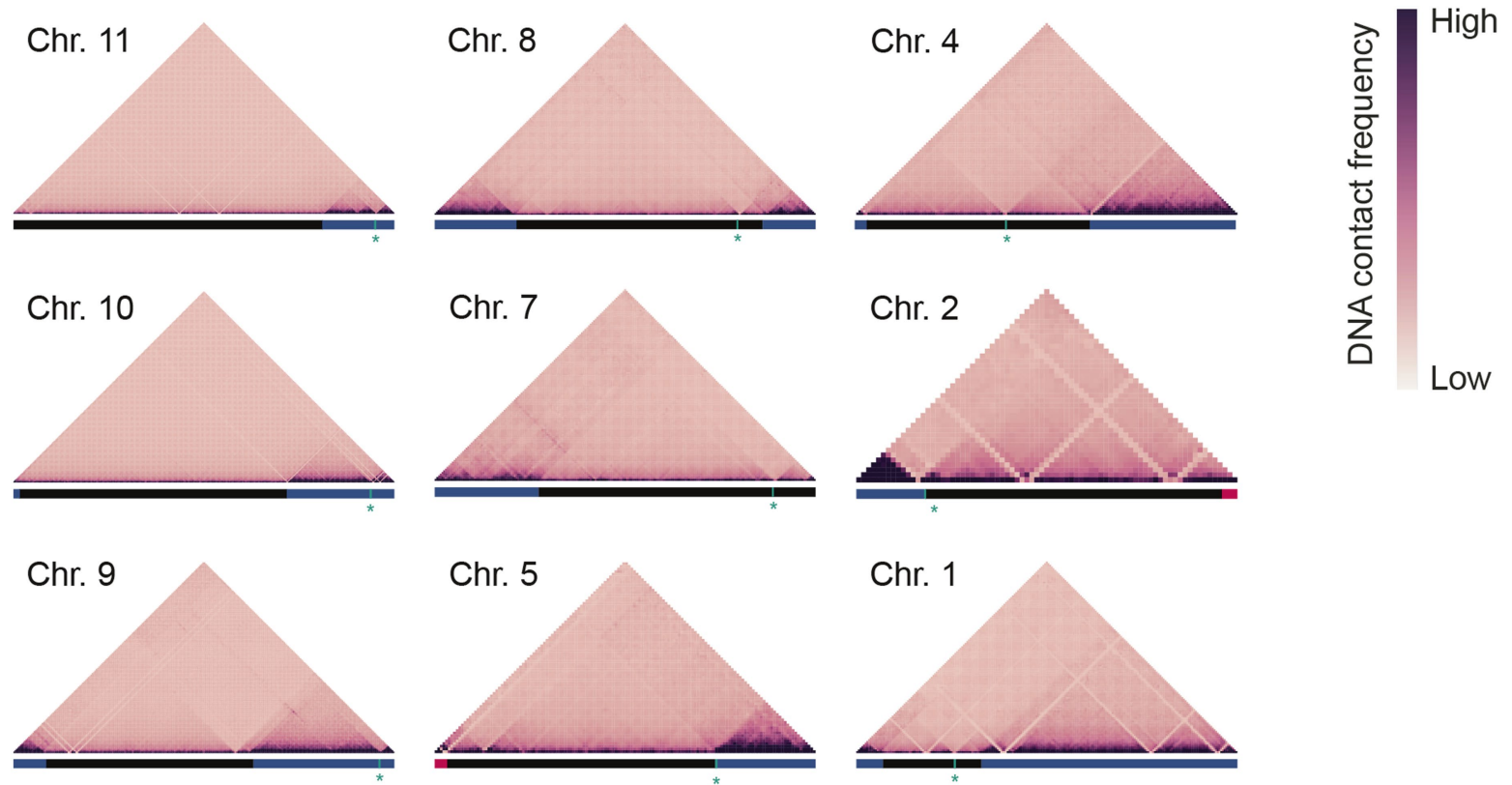

b

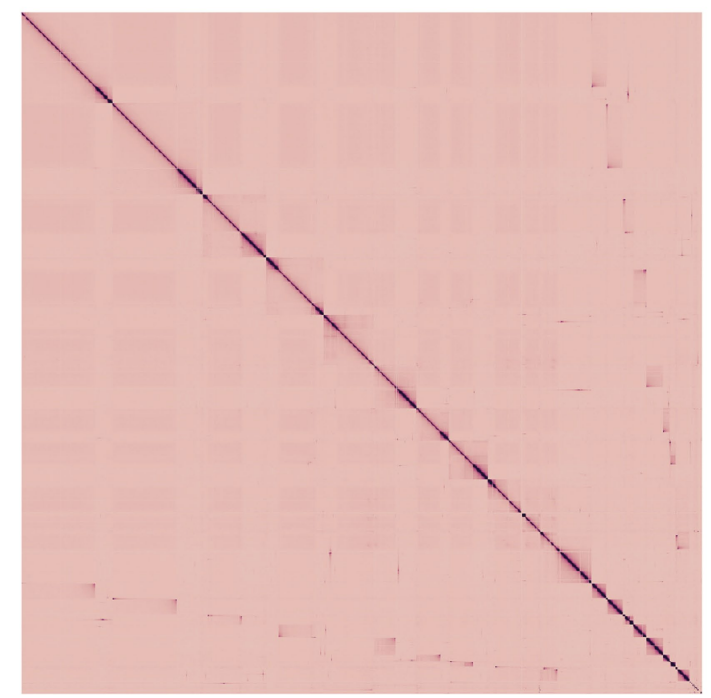

Extended Data Fig. 3 Compartmentalization of megabase chromosomes in wild-type cells. a, Hi-C heat maps of individual chromosomes at 20-kb resolution. Horizontal lines mark subtelomeric regions (blue), core regions (black) and bloodstream-form expression sites (red). A blue vertical line and an asterisk indicate the locations of centromeres. $\mathbf{b}, \mathrm{Hi}-\mathrm{C}$ heat map of the haploid genome with one set
C

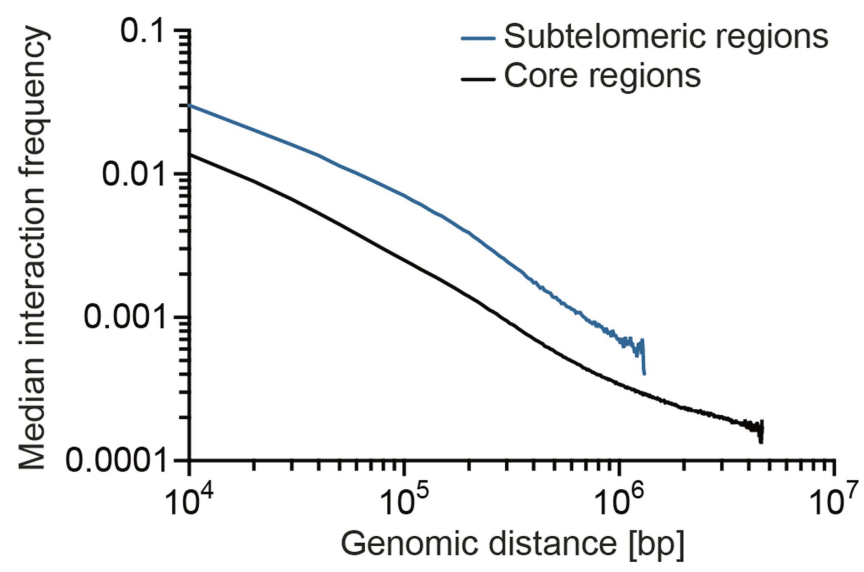

of subtelomeric regions joined to the core regions (20-kb resolution). c, Decay of frequency of intra-chromosomal contacts as a function of genomic distance (20-kb bin size) within subtelomeric (blue) and core (black) regions. The median across the core $(n=11)$ and subtelomeres $(n=32)$ is shown. 
a

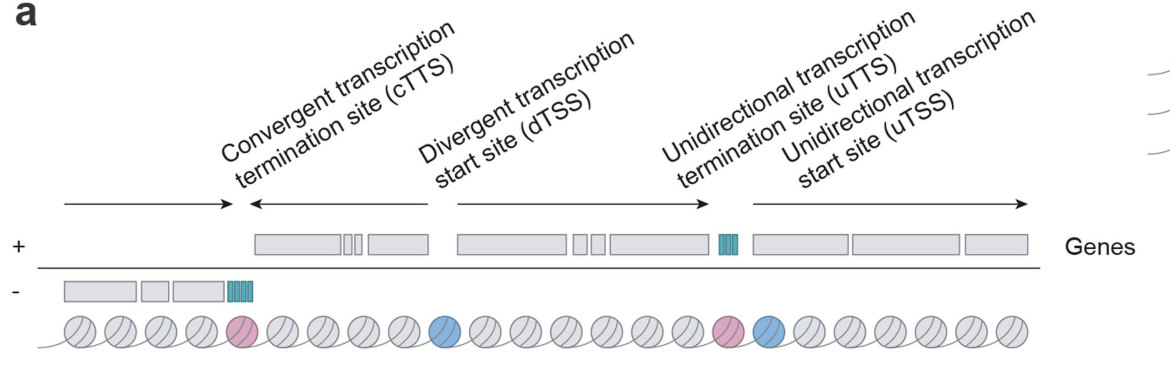

$\mathrm{H} 2 \mathrm{~A} / \mathrm{H} 2 \mathrm{~B} / \mathrm{H} 3 / \mathrm{H} 4$

$\mathrm{H} 2 \mathrm{~A} . \mathrm{Z} / \mathrm{H} 2 \mathrm{~B} . \mathrm{V} / \mathrm{H} 3 / \mathrm{H} 4$

$\mathrm{H} 2 \mathrm{~A} / \mathrm{H} 2 \mathrm{~B} / \mathrm{H} 3 . \mathrm{V} / \mathrm{H} 4 . \mathrm{V}$

tRNA or rRNA genes

b

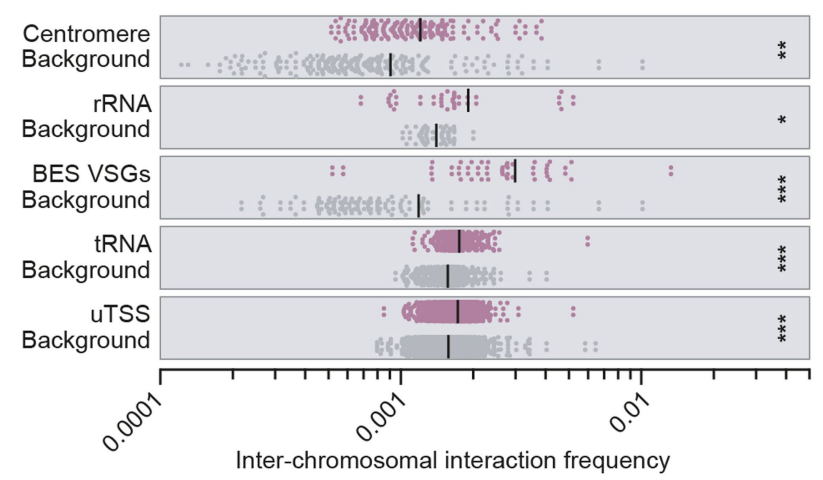

C

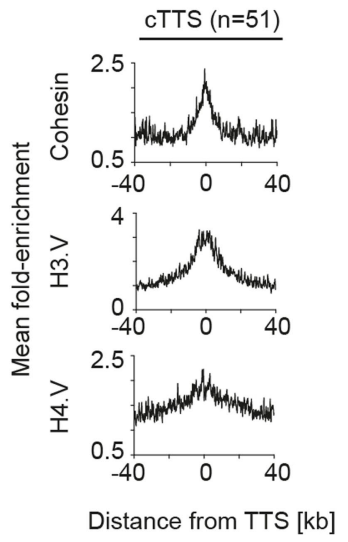

Extended Data Fig. $4 \mid \mathrm{Hi}-\mathrm{C}$ and ChIP-seq reveal partitioning of the T. brucei genome into distinct domains. a, Outline of the genome organization. Boundaries of transcription units are marked by nucleosomes that contain different types of histone variants. Black arrows indicate the direction of transcription. $\mathbf{b}$, Scatter plot showing inter-chromosomal interactions among centromeres $(n=206$ query bins, $n=292$ background bins, $P=0.0029)$, VSG genes ( $n=54$ query bins, $n=130$ background bins, $\left.P=1.63 \times 10^{-6}\right)$, rRNA genes $(n=40$ query bins, $n=64$ background bins, $P=0.0177)$, tRNA genes ( $n=614$ query bins, $n=620$ background bins, $\left.P=2.45 \times 10^{-190}\right)$ and unidirectional transcription start sites ( $n=3,142$ query bins, $n=3,682$ background bins, $P=6.49 \times 10^{-91}$ ) compared to a background sample, which was randomly selected from the interaction matrix ( $50-\mathrm{kb}$ bin size). The background sample matches the genomic feature in size and number. Selected bins with zero values were removed from both the query and background sample. $P$ values are based on Welch's $t$-test (two-sided). Black lines represent the mean. c, ChIP-seq data showing cohesin, H3.V and H4.V enrichment (compared to input material) averaged across all convergent transcription termination sites (cTTS, $n=51$ ) (window size, $101 \mathrm{bp}$; step size, $11 \mathrm{bp}$ ). 
a

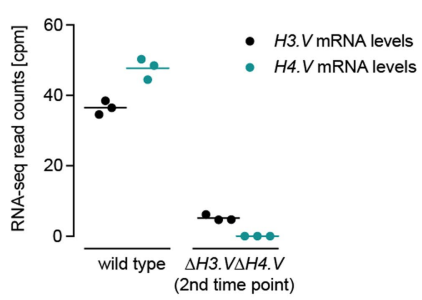

C

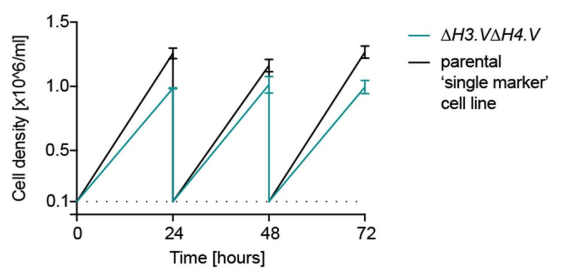

b
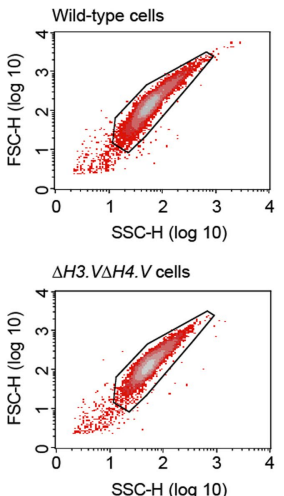

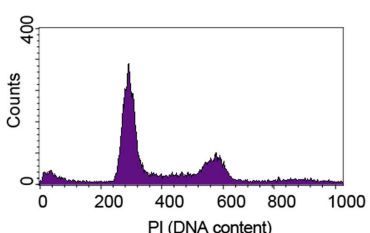

$\mathrm{PI}$ (DNA content)

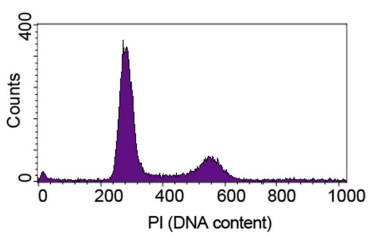

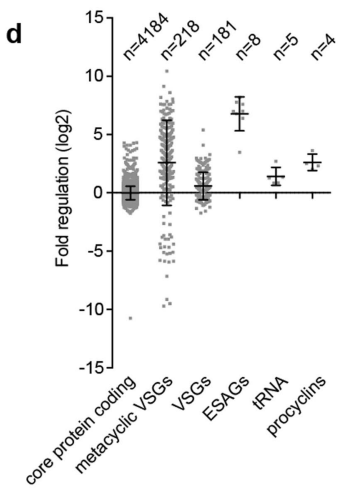

Extended Data Fig. $5 \mid$ Characterization of $\Delta H 3 . V \Delta H 4 . V$ cells.

a, RNA-seq fragment counts on H3.V and H4.V CDS in wild-type and $\Delta H 3 . V \Delta H 4 . V$ cells, normalized by million fragments mapped to proteincoding genes. Note that the first and last codon of the $H 3$. $V$ open reading frame were not deleted. As a result, a small number of $H 3 . V$ reads are detected even in the $\Delta H 3$. $V$ cells. $\mathbf{b}$, Cell-cycle analysis based on flow cytometry, of wild-type and $\Delta H 3 . V \Delta H 4 . V$ cells. One of three replicates is shown. c, Growth curve (mean \pm s.d.) of wild-type and $\Delta H 3 . V \Delta H 4 . V$ cells ( $n=3$ biologically independent replicates). d, RNA-seq of $\Delta H 3 . V \Delta H 4 . V$ cells (first and second time points). The mean \pm s.d. fold change in expression compared to wild type ( $n=3$ biologically independent experiments) is shown, for the significantly regulated genes (based on a Benjamini-Hochberg adjusted $P$ value from a two-sided Wald test with false discovery rate $<0.1)$ from different gene groups. ESAGs, expression-site-associated genes. 


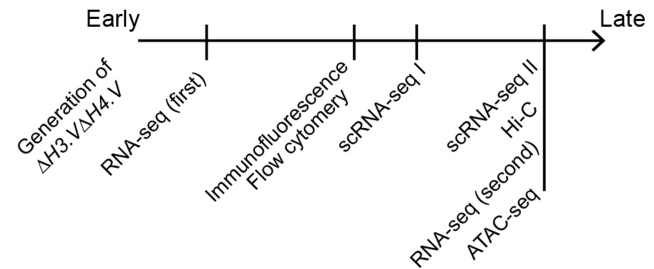

b

BES VSGs MES VSGs

BES VSGs

MES VSGS

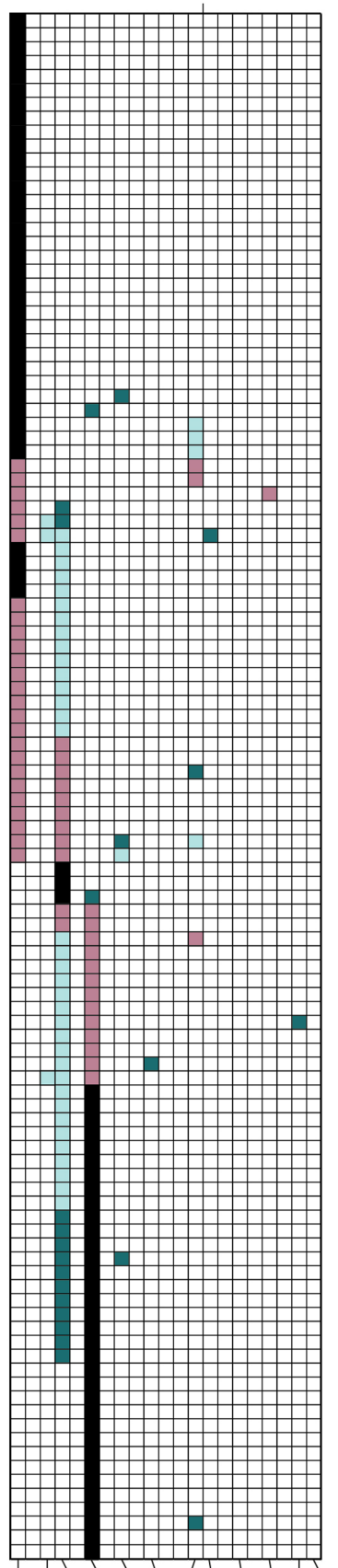

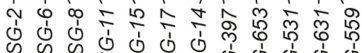

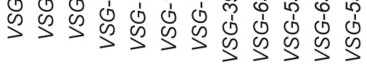

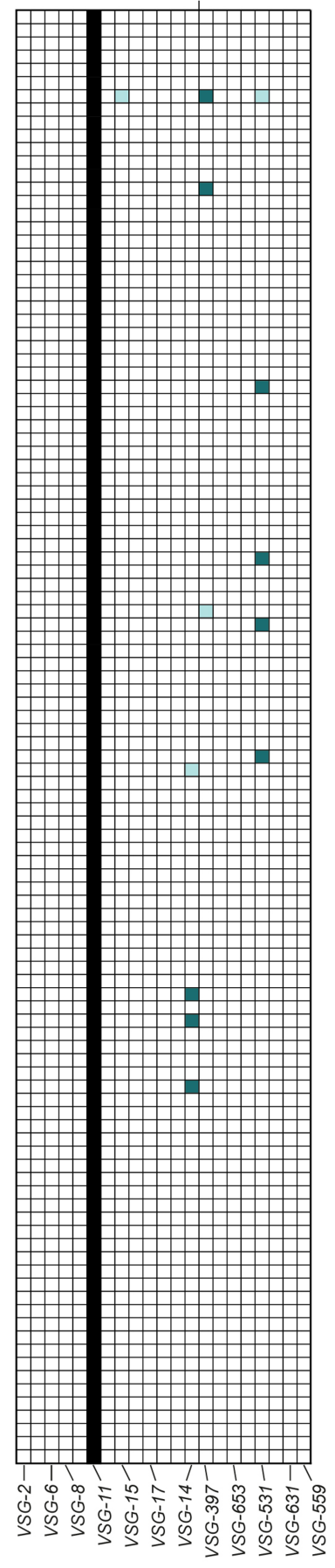

BES VSGs MES VSGs

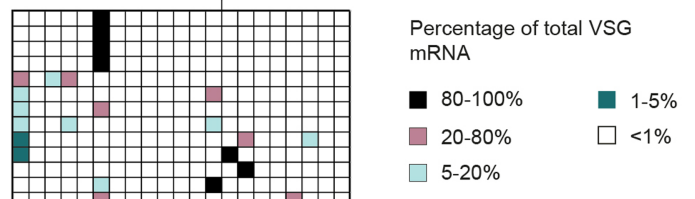

Extended Data Fig. $6 \mid$ Analysis of $\Delta H 3 . V \Delta H 4 . V$ cells. a, Order of cell analyses. b, scRNA-seq analyis of $\Delta H 3$. $V \Delta H 4 . V$ cells, at the second time point. $(n=338)$. Each row represents data from one cell. For details, see Fig. 3a, Extended Data Fig. 7. 
a

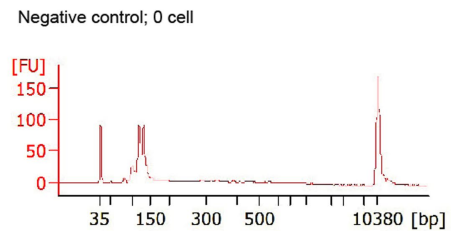

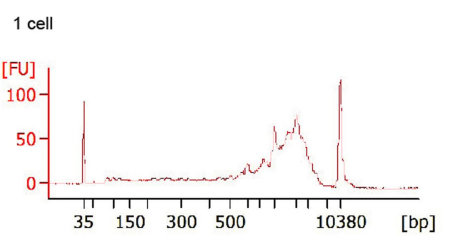

b

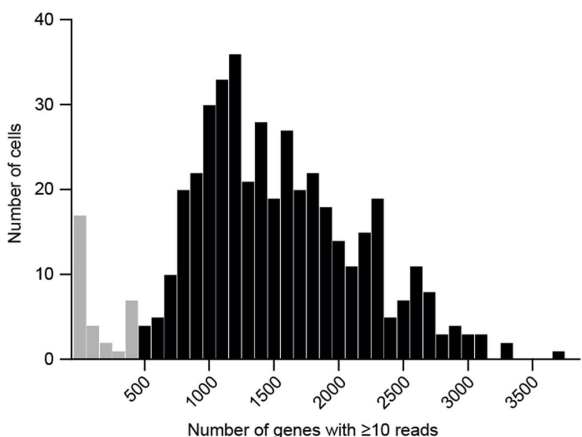

C Mapping uniquely
scRNAseq reads

Number of read counts

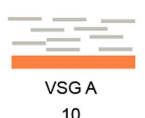

10

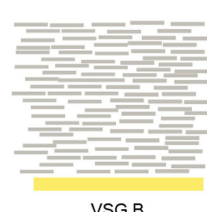
100

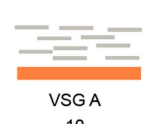

10

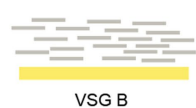

20
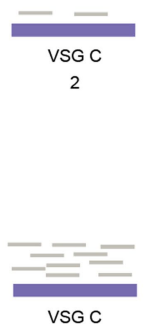

VSG C
Extended Data Fig. 7 | scRNA-seq quality control, VSG gene normalization and quantification. a, Representative Bioanalyzer profiles (Agilent) of cDNA from 0 cells $(n=6)$ and 1 cell $(n=18$, supplemented with ERCC spike-in control). b, Histogram representing the total number of genes expressed per single cell (wild type and $\Delta H 3 . V \Delta H 4 . V$; $n=452$ ). Cells with fewer than 500 genes (grey bars) were excluded from the analysis. c, Diagram representing quantification of expression of VSG genes, and the normalization procedure. The reads obtained in each single-cell library were mapped to the genome, keeping only the
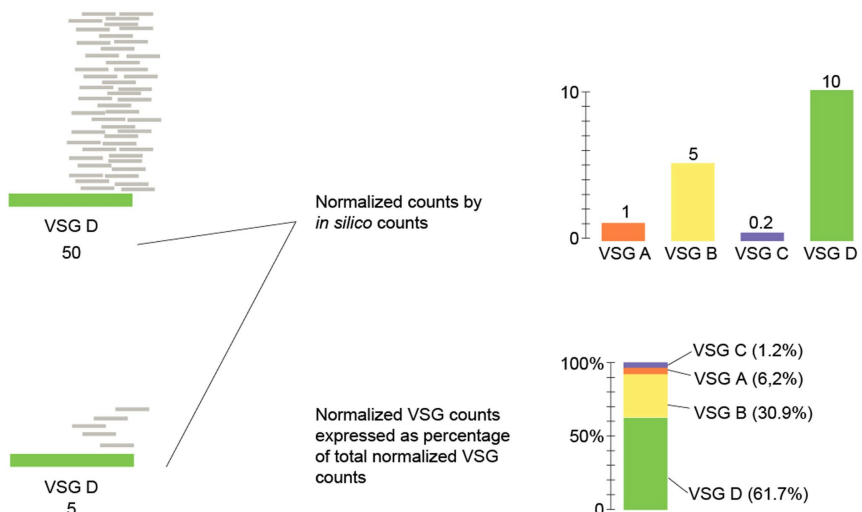

uniquely mapping reads (mapq $>0$ ). Next, the number of reads mapping to each VSG gene was quantified. To account for differences in length and 'uniqueness' among the different VSG genes, the same procedure was performed with an in silico set of reads. The read counts to each VSG gene in each scRNA-seq assay were normalized for 'uniqueness' and gene length by dividing them by the counts obtained with the in silico dataset. Finally, for each cell the normalized read counts for each VSG gene were expressed as a percentage of the total number of normalized counts to VSG genes. 

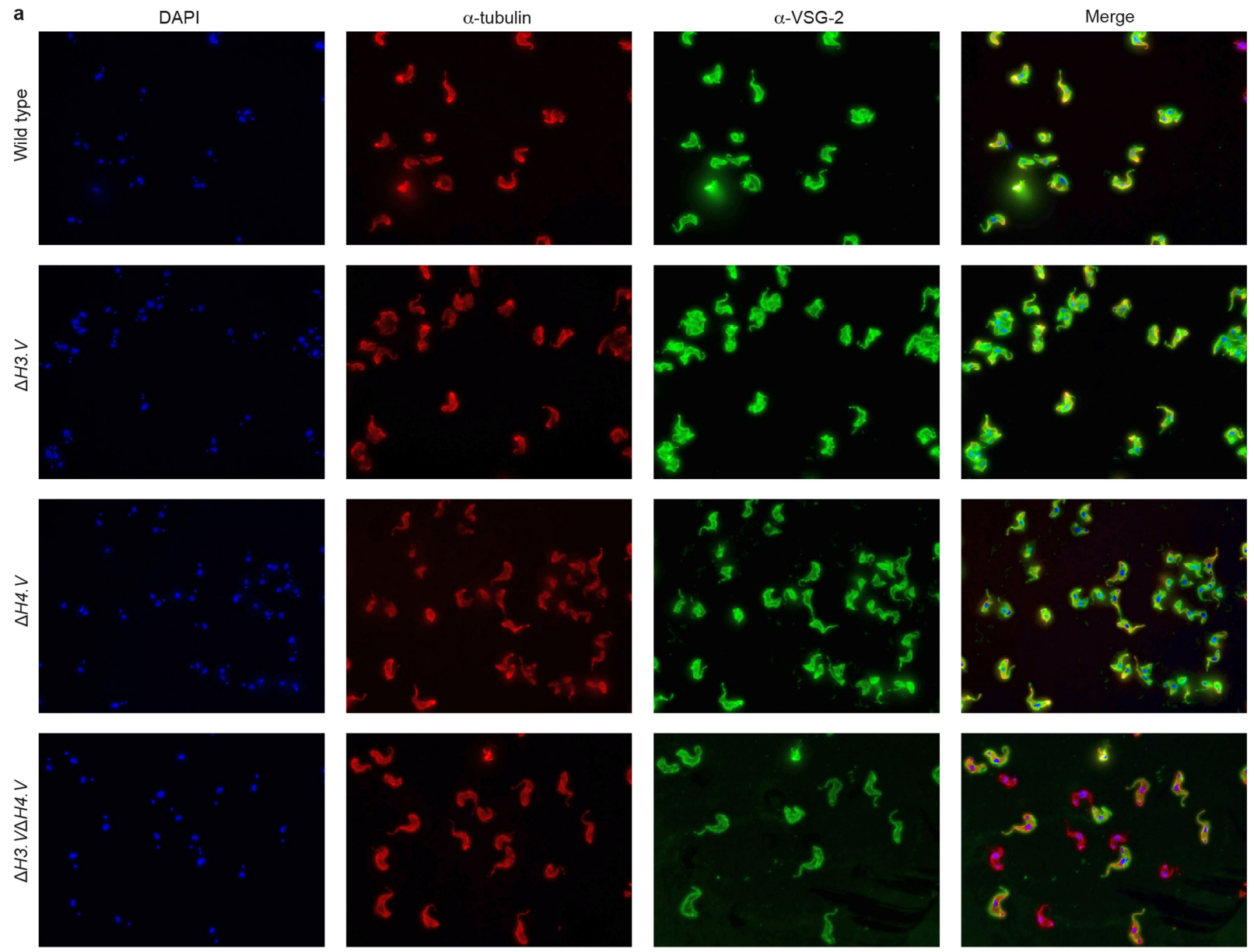

b
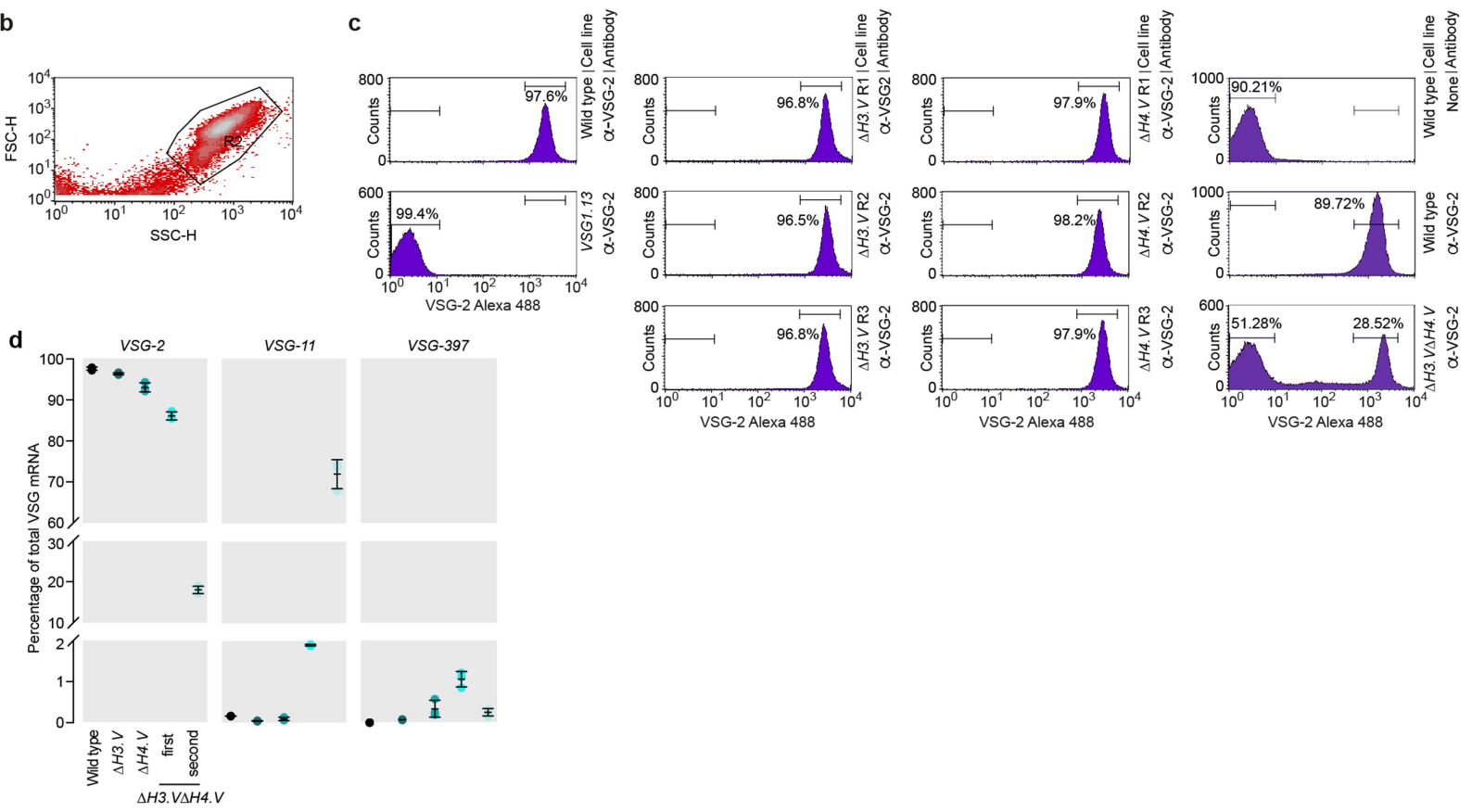

Extended Data Fig. $8 \mid$ Mutually exclusive expression of VSG genes is not lost in $\Delta H 3 . V$ and $\Delta H 4 . V$ single-knockout cells.

a, Immunofluorescence imaging in wild-type, $\Delta H 3 . V, \Delta H 4 . V$ and $\Delta H 3$. $V \Delta H 4 . V$ cells $(n=1)$. Representative images of $26-28$ stacks

$(0.1976-\mu \mathrm{m}$ voxel size, maximum projection) are shown. Scale bar, $10 \mu \mathrm{m}$. b, Gating strategy used for all analyses. c, Flow cytometry analysis of VSG2 expression in $\Delta H 3 . V, \Delta H 4 . V$ and $\Delta H 3 . V \Delta H 4 . V$ cells. Wild-type cells were used as a VSG-2 positive control, and cells expressing VSG-13 were used as a negative control. $\Delta H 3 . V, n=3 ; \Delta H 4 . V, n=3 ; \Delta H 3 . V \Delta H 4 . V$, $n=7$ (measured at different time points). For each assay, 50,000 events were gated. d, Heterogeneity in expression of VSG genes, based on RNAseq. The contributions of the dominant VSG-2 and two additional VSG genes found to be upregulated in $\Delta H 3 . V \Delta H 4 . V$ cells relative to the total VSG mRNAs are depicted. For each condition, mean mRNA levels and s.d. are derived from $n=3$ biologically independent RNA-seq experiments. 

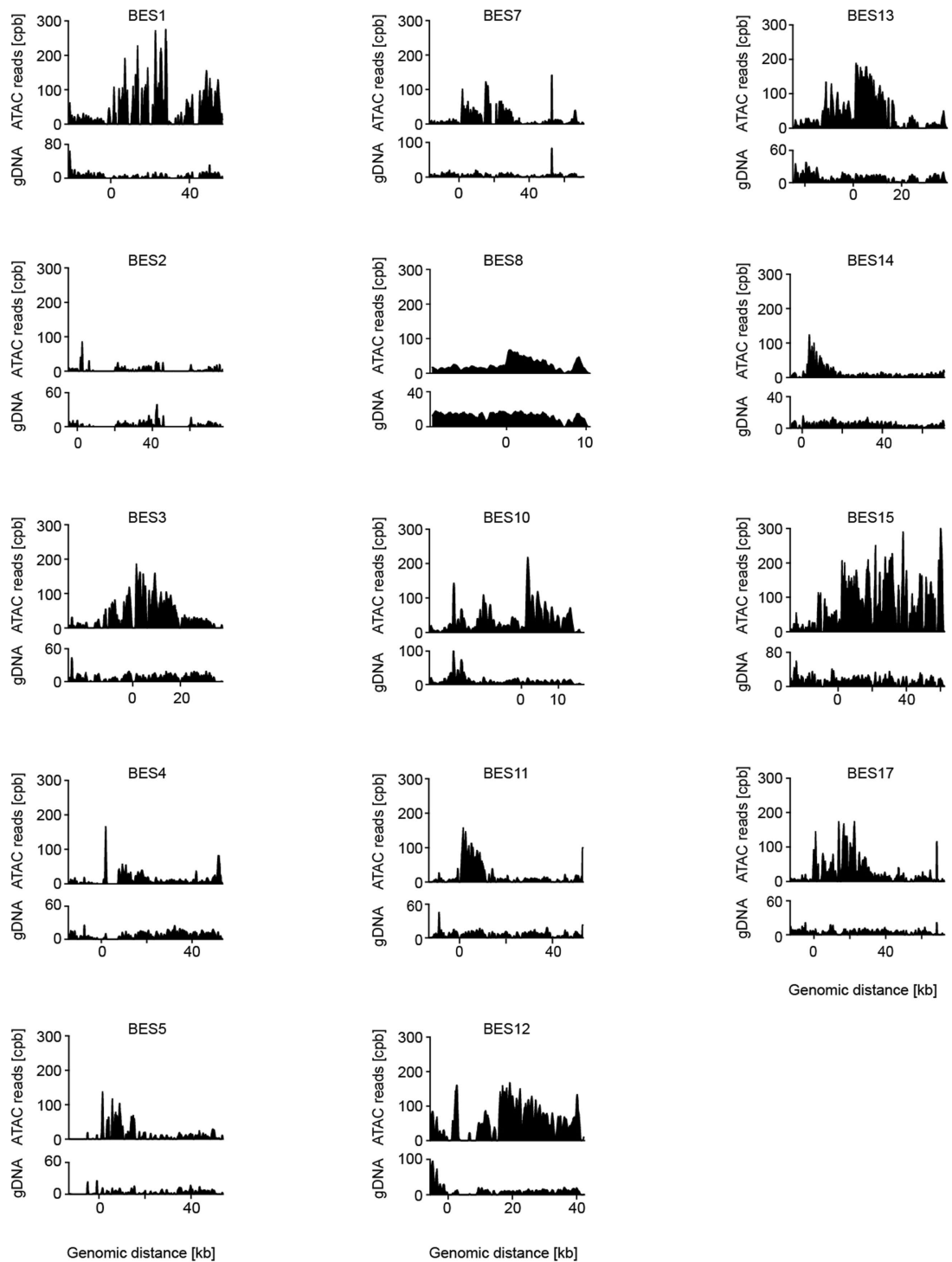

Genomic distance [kb]

. cells. Uniquely mapping ATAC-seq reads across all BESs are shown. The 0 -nt position corresponds to the promoter. Uniquely mapping gDNA-seq $n=2$ biologically independent experiments were performed, using sample material from 10 million cells in one experiment and from 20 million cells in the other.

reads are shown to illustrate differences in mappability. For ATAC-seq, 


\section{Extended Data Table 1 | Genome assembly statistics}

\begin{tabular}{ll}
\hline Parameter & Number \\
\hline \multicolumn{1}{c}{ The genome } \\
Total size (Mb) & 42.25 \\
Core regions (diploid) (Mb) & 22.71 \\
Subtelomeric region (haploid) (Mb) & 19.54 \\
Megabase chromosomes & 11 \\
Contigs & 96 \\
VSG expression sites assigned & 14 \\
to megabase chromosomes & \\
& \\
& \multicolumn{1}{c}{ The assembly } \\
SMRT reads & 642,583 \\
SMRT read N50 (kb) & 11.94 \\
Contigs after HGAPv3 assembly & 1232 \\
HGAPv3 contig N50 (kb) & 251 \\
Sequence assigned to megabase chr. (Mb) & 42.25 \\
Sequence assigned to mini- or & 0.8 \\
intermediate chromosomes (Mb) & 11.08 \\
Unscaffolded contigs (Mb) & \\
\hline
\end{tabular}

DNA elements that are smaller than $1 \mathrm{Mb}-$ for example, mini-chromosomes or circular DNA-were not scaffolded for this study, and contribute to the un-scaffolded contigs. 


\section{Reporting Summary}

Nature Research wishes to improve the reproducibility of the work that we publish. This form provides structure for consistency and transparency in reporting. For further information on Nature Research policies, see Authors \& Referees and the Editorial Policy Checklist.

\section{Statistical parameters}

When statistical analyses are reported, confirm that the following items are present in the relevant location (e.g. figure legend, table legend, main text, or Methods section).

n/a Confirmed

$\bigotimes$ The exact sample size $(n)$ for each experimental group/condition, given as a discrete number and unit of measurement

$\searrow$ An indication of whether measurements were taken from distinct samples or whether the same sample was measured repeatedly

$\triangle$ The statistical test(s) used AND whether they are one- or two-sided

Only common tests should be described solely by name; describe more complex techniques in the Methods section.

Х $\square$ A description of all covariates tested

Х $\square$ A description of any assumptions or corrections, such as tests of normality and adjustment for multiple comparisons

$\square$

A full description of the statistics including central tendency (e.g. means) or other basic estimates (e.g. regression coefficient) AND

variation (e.g. standard deviation) or associated estimates of uncertainty (e.g. confidence intervals)

$\triangle$ For null hypothesis testing, the test statistic (e.g. $F, t, r$ ) with confidence intervals, effect sizes, degrees of freedom and $P$ value noted

Give $P$ values as exact values whenever suitable.

Х $\square$ For Bayesian analysis, information on the choice of priors and Markov chain Monte Carlo settings

Х $\square$ For hierarchical and complex designs, identification of the appropriate level for tests and full reporting of outcomes

Х $\square$ Estimates of effect sizes (e.g. Cohen's $d$, Pearson's $r$ ), indicating how they were calculated

Clearly defined error bars

State explicitly what error bars represent (e.g. SD, SE, CI)

Our web collection on statistics for biologists may be useful.

\section{Software and code}

\section{Policy information about availability of computer code}

Data collection

no software for data collection was used

Data analysis

\section{Published code}

Cutadapt (version 1.15); Martin, M. et al. Cutadapt Removes Adapter Sequences From High-Throughput Sequencing Reads.

EMBnet.journal 17, 10-12 (2011)

bcl2fastq v.20.0.422

Fastx package (version 0.0.1.3)

HiCUP (version 0.5.9 devel); Wingett, S. et al. HiCUP: pipeline for mapping and processing Hi-C data. F1000Res 4, 1310 (2015)

$\mathrm{Hi}-\mathrm{C}$ pro (version 2.7.10); Servant, N. et al. HiC-Pro: an optimized and flexible pipeline for Hi-C data processing. Genome Biol 16, 259

(2015)

COVERnant (version 0.3.2); https://github.com/konrad/COVERnant

Samtools (version 1.8)

BWA-mem (version 0.7.12-r1039); https://arxiv.org/abs/1303.3997

SMRT Analysis (version 2.3.0); Chin, C. S. et al. Nonhybrid, finished microbial genome assemblies from long-read SMRT sequencing data. Nat Methods 10, 563-569 (2013)

PBJelly 2 (PBSuite_15.2.20); English, A. C. et al. Mind the gap: upgrading genomes with Pacific Biosciences RS long-read sequencing technology. PLoS One 7, e47768 (2012)

iCORN2; Otto, T. D., Sanders, M., Berriman, M. \& Newbold, C. Iterative Correction of Reference Nucleotides (iCORN) using second generation sequencing technology. Bioinformatics 26, 1704-1707 (2010) 
Companion; Steinbiss, S. et al. Companion: a web server for annotation and analysis of parasite genomes. Nucleic Acids Res 44, W29-34 (2016)

BEDTtools (version 2.26.0); Quinlan, A. R. \& Hall, I. M. BEDTools: a flexible suite of utilities for comparing genomic features.

Bioinformatics (2010)

DESeq2 (version 1.20.0); Love, M. I., Huber, W. \& Anders, S. Moderated estimation of fold change and dispersion for RNA-seq data with

DESeq2. Genome Biol 15, 550 (2014)

Seaborn (https://seaborn.pydata.org)

Fiji (https://fiji.sc/)

Imaris (version 8) (http://www.bitplane.com/imaris)

Custom code

The described analysis workflows and required custom made Unix Shell, Python and R scripts were deposited and are accessible at

Zenodo (DOI 10.5281/zenodo.823671). All data is available via NCBI GEO (accession GSM2586510) and EBI ENA (accession PRJEB18945).

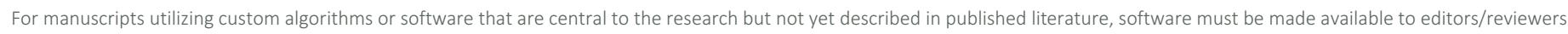

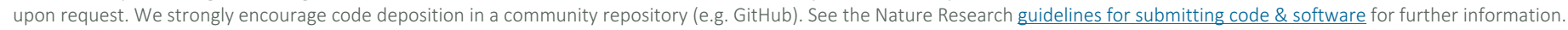

\section{Data}

Policy information about availability of data

All manuscripts must include a data availability statement. This statement should provide the following information, where applicable:

- Accession codes, unique identifiers, or web links for publicly available datasets

- A list of figures that have associated raw data

- A description of any restrictions on data availability

The RNA-seq, scRNA-seq, ChIP-seq, ATAC-seq and Hi-C sequencing data used in this publication have been deposited in NCBI's Gene Expression Omnibus69 and are accessible through GEO Series accession number GSE100896 (https://www.ncbi.nlm.nih.gov/geo/query/acc.cgi?acc=GSE100896). The raw SMRT sequencing reads and the genome assembly have been deposited in EBI's European Nucleotide Archive and are accessible through ENA Study accession number PRJEB18945 (http:// www.ebi.ac.uk/ena/data/view/PRJEB18945). Workflows and custom-made Unix Shell, Python and R scripts have been deposited at Zenodo (https://zenodo.org/ record/823671, https://doi.org/10.5281/zenodo.823671). Documentation to reproduce the data analysis is provided.

\section{Field-specific reporting}

Please select the best fit for your research. If you are not sure, read the appropriate sections before making your selection.

\Life sciences

Behavioural \& social sciences

Ecological, evolutionary \& environmental sciences

For a reference copy of the document with all sections, see nature.com/authors/policies/ReportingSummary-flat.pdf

\section{Life sciences study design}

All studies must disclose on these points even when the disclosure is negative.
Sample size
Sample sizes were not statistically predetermined.

Data exclusions

In the scRNA-seq analysis, only data from cells with more than 500 genes with more than 10 reads per gene were included. Therefore, data from 34 single cells were excluded because they were not matching the criteria.

Replication All attempts at replication were successful.

$\mathrm{Hi}-\mathrm{C}$ experiments were perfomed in triplicates for each cell line.

RNA-seq experiments were performed in triplicates for each isolate.

MNase-ChIP-seq experiments were performed in duplicates with an input control for each experiment.

Scc1-ChIP experiments were performed in triplicates with an input control for each experiment.

ATAC-seq experiments were performed in duplicates with different cell numbers, respectively. Two gDNA samples were included as internal

control for accessibility.

Single-cell RNA-seq analysis is based on 40 and 408 cells per cell line, respectively.

Flow cytometry experiments were performed in triplicates for each cell line.

Quantification of telomere clusters (FISH microscopy) was performed in duplicates (total number of cells: 1128).

Randomization Not relevant for this study as allocation of samples/organisms was not needed or intended.

Blinding Image acquisition and analysis was done in a blinded fashion.

a) For IF images, pictures were taken randomly from the slide without "searching" for a suitable area.

b) FISH images were taken and analyzed by an unbiased, external investigator. This person also chose representative images for the publication.

For other analyses investigators were not blinded. 


\section{Reporting for specific materials, systems and methods}

Materials \& experimental systems

\begin{tabular}{l|l}
\hline n/a & Involved in the study \\
$\square$ Unique biological materials \\
$\square$
\end{tabular}

\begin{tabular}{l|l}
\multicolumn{2}{l}{ Methods } \\
\hline n/a & Involved in the study \\
\hline & $\bigotimes$ ChIP-seq \\
$\square$ & $\square$ Flow cytometry
\end{tabular}

\section{Antibodies}

Antibodies used

Validation

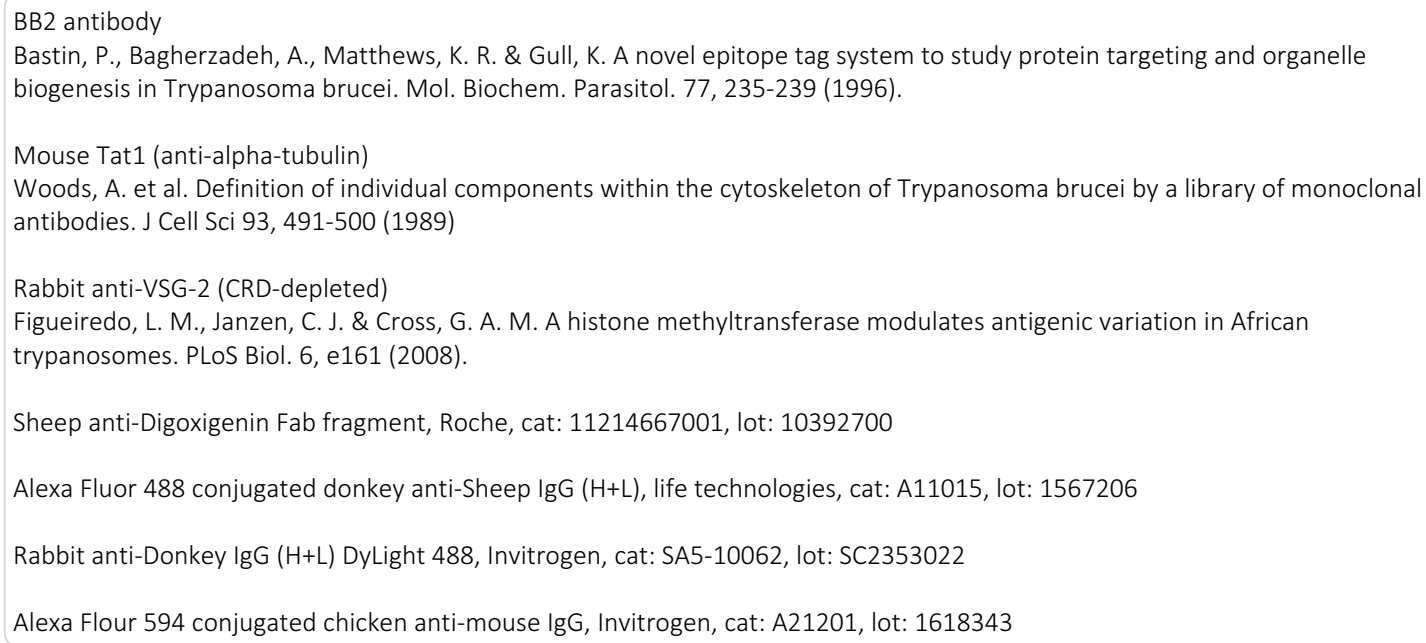

Х Confirm that both raw and final processed data have been deposited in a public database such as GEO.

Х Confirm that you have deposited or provided access to graph files (e.g. BED files) for the called peaks.

Data access links

May remain private before publication.

Files in database submission
GEO Series accession number GSE100896 (https://www.ncbi.nlm.nih.gov/geo/query/acc.cgi?acc=GSE100896)

\section{H3V ChIP Raw}

L1800150_H3_V_Ty_MNase_ChIP_1_R1.fq.gz

L1800150_H3_V_Ty_MNase_ChIP_1_R2.fq.gz

L1800151_H3_V_Ty_MNase_Input_1_R1.fq.gz

L1800151_H3_V_Ty_MNase_Input_1_R2.fq.gz

L1800152_H3_V_Ty_MNase_ChIP_2_R1.fq.gz 
L1800152_H3_V_Ty_MNase_ChIP_2_R2.fq.gz

L1800153_H3_V_Ty_MNase_Input_2_R1.fq.gz

L1800153_H3_V_Ty_MNase_Input_2_R2.fq.gz

Output - H3V ChIP

alignment

L1800150_H3_V_Ty_MNase_ChIP_1_to_HGAP3_Tb427v9_bwa.sorted.bam

L1800150_H3_V_Ty_MNase_ChIP_1_to_HGAP3_Tb427v9_bwa.sorted.bam.bai

L1800150_H3_V_Ty_MNase_ChIP_1_to_HGAP3_Tb427v9_bwa.sorted.mapq10.bam

L1800150_H3_V_Ty_MNase_ChIP_1_to_HGAP3_Tb427v9_bwa.sorted.mapq10.bam.bai

L1800151_H3_V_Ty_MNase_Input_1_to_HGAP3_Tb427v9_bwa.sorted.bam

L1800151_H3_V_Ty_MNase_Input_1_to_HGAP3_Tb427v9_bwa.sorted.bam.bai

L1800151_H3_V_Ty_MNase_Input_1_to_HGAP3_Tb427v9_bwa.sorted.mapq10.bam

L1800151_H3_V_Ty_MNase_Input_1_to_HGAP3_Tb427v9_bwa.sorted.mapq10.bam.bai

L1800152_H3_V_Ty_MNase_ChIP_2_to_HGAP3_Tb427v9_bwa.sorted.bam

L1800152_H3_V_TY_MNase_ChIP_2_to_HGAP3_Tb427v9_bwa.sorted.bam.bai

L1800152_H3_V_Ty_MNase_ChIP_2_to_HGAP3_Tb427v9_bwa.sorted.mapq10.bam

L1800152_H3_V_Ty_MNase_ChIP_2_to_HGAP3_Tb427v9_bwa.sorted.mapq10.bam.bai

L1800153_H3_V_Ty_MNase_Input_2_to_HGAP3_Tb427v9_bwa.sorted.bam

L1800153_H3_V_Ty_MNase_Input_2_to_HGAP3_Tb427v9_bwa.sorted.bam.bai

L1800153_H3_V_Ty_MNase_Input_2_to_HGAP3_Tb427v9_bwa.sorted.mapq10.bam

L1800153_H3_V_Ty_MNase_Input_2_to_HGAP3_Tb427v9_bwa.sorted.mapq10.bam.bai

H3V ChIP Wiggle files (coverage plots)

ws1ss1_denominator_L1800151_H3_V_Ty_MNase_Input_1_to_HGAP3_Tb427v9_bwa.sorted.mapq10.wig ws1ss1_denominator_L1800153_H3_V_Ty_MNase_Input_2_to_HGAP3_Tb427v9_bwa.sorted.mapq10.wig ws1ss1_numerator_L1800150_H3_V_Ty_MNase_ChIP_1_to_HGAP3_Tb427v9_bwa.sorted.mapq10.wig ws1ss1_numerator_L1800152_H3_V_Ty_MNase_ChIP_2_to_HGAP3_Tb427v9_bwa.sorted.mapq10.wig ws1ss1_ratio_L1800151_H3_V_Ty_MNase_Input_1_to_HGAP3_Tb427v9_bwa.sorted.mapq10_vs_L1800150_H3_V_Ty_MN ase_ChIP_1_to_HGAP3_Tb427v9_bwa.sorted.mapq10.wig

ws1ss1_ratio_L1800153_H3_V_Ty_MNase_Input_2_to_HGAP3_Tb427v9_bwa.sorted.mapq10_Vs_L1800152_H3_V_Ty_MN ase_ChIP_2_to_HGAP3_Tb427v9_bwa.sorted.mapq10.wig

ws2001ss501_denominator_L1800151_H3_V_Ty_MNase_Input_1_to_HGAP3_Tb427v9_bwa.sorted.mapq10.wig ws2001ss501_denominator_L1800153_H3_V_Ty_MNase_Input_2_to_HGAP3_Tb427v9_bwa.sorted.mapq10.wig ws2001ss501_numerator_L1800150_H3_V_Ty_MNase_ChIP_1_to_HGAP3_Tb427v9_bwa.sorted.mapq10.wi ws2001ss501_numerator_L1800152_H3_V_Ty_MNase_ChIP_2_to_HGAP3_Tb427v9_bwa.sorted.mapq10.wi ws2001ss501_ratio_L1800151_H3_V_TY_MNase_Input_1_to_HGAP3_Tb427v9_bwa.sorted.mapq10_Vs_L1800150_H3_V_T Y_MNase_ChIP_1_to_HGAP3_Tb427v9_bwa.sorted.mapq10.wig

ws2001ss501_ratio_L1800153_H3_V_Ty_MNase_Input_2_to_HGAP3_Tb427v9_bwa.sorted.mapq10_vs_L1800152_H3_V_T y_MNase_ChIP_2_to_HGAP3_Tb427v9_bwa.sorted.mapq10.wig

ws501ss101_denominator_L1800151_H3_V_Ty_MNase_Input_1_to_HGAP3_Tb427v9_bwa.sorted.mapq10.wig ws501ss101_denominator_L1800153_H3_V_Ty_MNase_Input_2_to_HGAP3_Tb427v9_bwa.sorted.mapq10.wig ws501ss101_numerator_L1800150_H3_V_Ty_MNase_ChIP_1_to_HGAP3_Tb427v9_bwa.sorted.mapq10.wig ws501ss101_numerator_L1800152_H3_V_Ty_MNase_ChIP_2_to_HGAP3_Tb427v9_bwa.sorted.mapq10.wig ws501ss101_ratio_L1800151_H3_V_Ty_MNase_Input_1_to_HGAP3_Tb427v9_bwa.sorted.mapq10_vs_L1800150_H3_V_Ty _MNase_ChIP_1_to_HGAP3_Tb427v9_bwa.sorted.mapq10.wig

ws501ss101_ratio_L1800153_H3_V_Ty_MNase_Input_2_to_HGAP3_Tb427v9_bwa.sorted.mapq10_Vs_L1800152_H3_V_Ty _MNase_ChIP_2_to_HGAP3_Tb427v9_bwa.sorted.mapq10.wig

H4V ChIP Raw

L1800154_H4_V_Ty_MNase_ChIP_1_R1.fq.gz

L1800154_H4_V_Ty_MNase_ChIP_1_R2.fq.gz

L1800155_H4_V_Ty_MNase_Input_1_R1.fq.gz

L1800155_H4_V_Ty_MNase_Input_1_R2.fq.gz

L1800156_H4_V_Ty_MNase_ChIP_2_R1.fq.gz

L1800156_H4_V_Ty_MNase_ChIP_2_R2.fq.gz

L1800157_H4_V_Ty_MNase_Input_2_R1.fq.gz

L1800157_H4_V_Ty_MNase_Input_2_R2.fq.gz

Output H4V ChIP

Alignment

L1800154_H4_V_Ty_MNase_ChIP_1_to_HGAP3_Tb427v9_bwa.sorted.bam

L1800154_H4_V_TY_MNase_ChIP_1_to_HGAP3_Tb427v9_bwa.sorted.bam.bai

L1800154_H4_V_Ty_MNase_ChIP_1_to_HGAP3_Tb427v9_bwa.sorted.mapq10.bam

L1800154_H4_V_Ty_MNase_ChIP_1_to_HGAP3_Tb427v9_bwa.sorted.mapq10.bam.bai

L1800155_H4_V_Ty_MNase_Input_1_to_HGAP3_Tb427v9_bwa.sorted.bam

L1800155_H4_V_Ty_MNase_Input_1_to_HGAP3_Tb427v9_bwa.sorted.bam.bai

L1800155_H4_V_Ty_MNase_Input_1_to_HGAP3_Tb427v9_bwa.sorted.mapq10.bam

L1800155_H4_V_Ty_MNase_Input_1_to_HGAP3_Tb427v9_bwa.sorted.mapq10.bam.bai

L1800156_H4_V_Ty_MNase_ChIP_2_to_HGAP3_Tb427v9_bwa.sorted.bam

L1800156_H4_V_Ty_MNase_ChIP_2_to_HGAP3_Tb427v9_bwa.sorted.bam.bai

L1800156_H4_V_Ty_MNase_ChIP_2_to_HGAP3_Tb427v9_bwa.sorted.mapq10.bam

L1800156_H4_V_Ty_MNase_ChIP_2_to_HGAP3_Tb427v9_bwa.sorted.mapq10.bam.bai

L1800157_H4_V_Ty_MNase_Input_2_to_HGAP3_Tb427v9_bwa.sorted.bam

L1800157_H4_V_Ty_MNase_Input_2_to_HGAP3_Tb427v9_bwa.sorted.bam.bai 
L1800157_H4_V_Ty_MNase_Input_2_to_HGAP3_Tb427v9_bwa.sorted.mapq10.bam L1800157_H4_V_Ty_MNase_Input_2_to_HGAP3_Tb427v9_bwa.sorted.mapq10.bam.bai

H4V ChIPs Wiggle files (coverage plots)

ws1ss1_denominator_L1800155_H4_V_Ty_MNase_Input_1_to_HGAP3_Tb427v9_bwa.sorted.mapq10.wig ws1ss1_denominator_L1800157_H4_V_Ty_MNase_Input_2_to_HGAP3_Tb427v9_bwa.sorted.mapq10.wig ws1ss1_numerator_L1800154_H4_V_Ty_MNase_ChIP_1_to_HGAP3_Tb427v9_bwa.sorted.mapq10.wig ws1ss1_numerator_L1800156_H4_V_Ty_MNase_ChIP_2_to_HGAP3_Tb427v9_bwa.sorted.mapq10.wig ws1ss1_ratio_L1800155_H4_V_Ty_MNase_Input_1_to_HGAP3_Tb427v9_bwa.sorted.mapq10_vs_L1800154_H4_V_Ty_MN ase_ChIP_1_to_HGAP3_Tb427v9_bwa.sorted.mapq10.wig

ws1ss1_ratio_L1800157_H4_V_Ty_MNase_Input_2_to_HGAP3_Tb427v9_bwa.sorted.mapq10_vs_L1800156_H4_V_Ty_MN ase_ChIP_2_to_HGAP3_Tb427v9_bwa.sorted.mapq10.wig

ws2001ss501_denominator_L1800155_H4_V_Ty_MNase_Input_1_to_HGAP3_Tb427v9_bwa.sorted.mapq10.wig ws2001ss501_denominator_L1800157_H4_V_Ty_MNase_Input_2_to_HGAP3_Tb427v9_bwa.sorted.mapq10.wig ws2001ss501_numerator_L1800154_H4_V_Ty_MNase_ChIP_1_to_HGAP3_Tb427v9_bwa.sorted.mapq10.wig ws2001ss501_numerator_L1800156_H4_V_Ty_MNase_ChIP_2_to_HGAP3_Tb427v9_bwa.sorted.mapq10.wig ws2001ss501_ratio_L1800155_H4_V_Ty_MNase_Input_1_to_HGAP3_Tb427v9_bwa.sorted.mapq10_Vs_L1800154_H4_V_T Y_MNase_ChIP_1_to_HGAP3_Tb427v9_bwa.sorted.mapq10.wig

ws2001ss501_ratio_L1800157_H4_V_Ty_MNase_Input_2_to_HGAP3_Tb427v9_bwa.sorted.mapq10_Vs_L1800156_H4_V_T y_MNase_ChIP_2_to_HGAP3_Tb427v9_bwa.sorted.mapq10.wig

ws501ss101_denominator_L1800155_H4_V_Ty_MNase_Input_1_to_HGAP3_Tb427v9_bwa.sorted.mapq10.wig ws501ss101_denominator_L1800157_H4_V_Ty_MNase_Input_2_to_HGAP3_Tb427v9_bwa.sorted.mapq10.wig ws501ss101_numerator_L1800154_H4_V_Ty_MNase_ChIP_1_to_HGAP3_Tb427v9_bwa.sorted.mapq10.wig ws501ss101_numerator_L1800156_H4_V_Ty_MNase_ChIP_2_to_HGAP3_Tb427v9_bwa.sorted.mapq10.wig ws501ss101_ratio_L1800155_H4_V_Ty_MNase_Input_1_to_HGAP3_Tb427v9_bwa.sorted.mapq10_Vs_L1800154_H4_V_Ty _MNase_ChIP_1_to_HGAP3_Tb427v9_bwa.sorted.mapq10.wig

ws501ss101_ratio_L1800157_H4_V_Ty_MNase_Input_2_to_HGAP3_Tb427v9_bwa.sorted.mapq10_vs_L1800156_H4_V_Ty _MNase_ChIP_2_to_HGAP3_Tb427v9_bwa.sorted.mapq10.wig

Scc1 ChIP Raw

L1800343_Scc1_Ty1_wt_soni_ChIP_0_R1.fq.gz

L1800343_Scc1_Ty1_wt_soni_ChIP_0_R2.fq.gz

L1800344_Scc1_Ty1_wt_soni_Input_0_R1.fq.gz

L1800344_Scc1_Ty1_wt_soni_Input_0_R2.fq.gz

L1800468_Scc1_Ty1_wt_soni_ChIP_1_R1.fq.gz

L1800468_Scc1_Ty1_wt_soni_ChIP_1_R2.fq.gz

L1800469_Scc1_Ty1_wt_soni_Input_1_R1.fq.gz L1800469_Scc1_Ty1_wt_soni_Input_1_R2.fq.gz L1800474_Scc1_Ty1_wt_soni_ChIP_2_R1.fa.gz L1800474_Scc1_Ty1_wt_soni_ChIP_2_R2.fa.gz L1800475_Scc1_Ty1_wt_soni_Input_2_R1.fq.gz L1800475_Scc1_Ty1_wt_soni_Input_2_R2.fq.gz

Output Scc1 ChIP

Alignment

L1800343_Scc1_Ty1_wt_soni_ChIP_0_to_HGAP3_Tb427v9_bwa.sorted.bam

L1800343_Scc1_Ty1_wt_soni_ChIP_0_to_HGAP3_Tb427v9_bwa.sorted.bam.bai

L1800343_Scc1_Ty1_wt_soni_ChIP_0_to_HGAP3_Tb427v9_bwa.sorted.mapq10.bam

L1800343_Scc1_Ty1_wt_soni_ChIP_0_to_HGAP3_Tb427v9_bwa.sorted.mapq10.bam.bai

L1800344_Scc1_Ty1_wt_soni_Input_0_to_HGAP3_Tb427v9_bwa.sorted.bam

L1800344_Scc1_Ty1_wt_soni_Input_0_to_HGAP3_Tb427v9_bwa.sorted.bam.bai

L1800344_Scc1_Ty1_wt_soni_Input_0_to_HGAP3_Tb427v9_bwa.sorted.mapq10.bam

L1800344_Scc1_Ty1_wt_soni_Input_0_to_HGAP3_Tb427v9_bwa.sorted.mapq10.bam.bai

L1800468_Scc1_Ty1_wt_soni_ChIP_1_to_HGAP3_Tb427v9_bwa.sorted.bam

L1800468_Scc1_Ty1_wt_soni_ChIP_1_to_HGAP3_Tb427v9_bwa.sorted.bam.bai

L1800468_Scc1_Ty1_wt_soni_ChIP_1_to_HGAP3_Tb427v9_bwa.sorted.mapq10.bam

L1800468_Scc1_Ty1_wt_soni_ChIP_1_to_HGAP3_Tb427v9_bwa.sorted.mapq10.bam.bai

L1800469_Scc1_Ty1_wt_soni_Input_1_to_HGAP3_Tb427v9_bwa.sorted.bam

L1800469_Scc1_Ty1_wt_soni_Input_1_to_HGAP3_Tb427v9_bwa.sorted.bam.bai

L1800469_Scc1_Ty1_wt_soni_Input_1_to_HGAP3_Tb427v9_bwa.sorted.mapq10.bam

L1800469_Scc1_Ty1_wt_soni_Input_1_to_HGAP3_Tb427v9_bwa.sorted.mapq10.bam.bai

L1800474_Scc1_Ty1_wt_soni_ChIP_2_to_HGAP3_Tb427v9_bwa.sorted.bam

L1800474_Scc1_Ty1_wt_soni_ChIP_2_to_HGAP3_Tb427v9_bwa.sorted.bam.bai

L1800474_Scc1_Ty1_wt_soni_ChIP_2_to_HGAP3_Tb427v9_bwa.sorted.mapq10.bam

L1800474_Scc1_Ty1_wt_soni_ChIP_2_to_HGAP3_Tb427v9_bwa.sorted.mapq10.bam.bai

L1800475_Scc1_Ty1_wt_soni_Input_2_to_HGAP3_Tb427v9_bwa.sorted.bam

L1800475_Scc1_Ty1_wt_soni_Input_2_to_HGAP3_Tb427v9_bwa.sorted.bam.bai

L1800475_Scc1_Ty1_wt_soni_Input_2_to_HGAP3_Tb427v9_bwa.sorted.mapq10.bam

L1800475_Scc1_Ty1_wt_soni_Input_2_to_HGAP3_Tb427v9_bwa.sorted.mapq10.bam.bai

Wiggle files Scc1 ChIP (coverage plots)

ws1ss1_denominator_L1800344_Scc1_Ty1_wt_soni_Input_0_to_HGAP3_Tb427v9_bwa.sorted.mapq10.wig ws1ss1_denominator_L1800469_Scc1_Ty1_wt_soni_Input_1_to_HGAP3_Tb427v9_bwa.sorted.mapq10.wig ws1ss1_denominator_L1800475_Scc1_Ty1_wt_soni_Input_2_to_HGAP3_Tb427v9_bwa.sorted.mapq10.wig ws1ss1_numerator_L1800343_Scc1_Ty1_wt_soni_ChIP_0_to_HGAP3_Tb427v9_bwa.sorted.mapq10.wig 
ws1ss1_numerator_L1800468_Scc1_Ty1_wt_soni_ChIP_1_to_HGAP3_Tb427v9_bwa.sorted.mapq10.wig ws1ss1 numerator L1800474 Scc1 Ty1 wt soni ChIP 2 to HGAP3 Tb427v9 bwa.sorted.mapq10.wig

ws1ss1_ratio_L1800344_Scc1_Ty1_wt_soni_Input_0_to_HGAP3_Tb427v9_bwa.sorted.mapq10_vs_L1800343_Scc1_Ty1_wt _soni_ChIP_0_to_HGAP3_Tb427v9_bwa.sorted.mapq10.wig

ws1ss1_ratio_L1800469_Scc1_Ty1_wt_soni_Input_1_to_HGAP3_Tb427v9_bwa.sorted.mapq10_vs_L1800468_Scc1_Ty1_wt _soni_ChIP_1_to_HGAP3_Tb427v9_bwa.sorted.mapq10.wig

ws1ss1_ratio_L1800475_Scc1_Ty1_wt_soni_Input_2_to_HGAP3_Tb427v9_bwa.sorted.mapq10_vs_L1800474_Scc1_Ty1_wt _soni_ChIP_2_to_HGAP3_Tb427v9_bwa.sorted.mapq10.wig

ws2001ss501_denominator_L1800344_Scc1_Ty1_wt_soni_Input_0_to_HGAP3_Tb427v9_bwa.sorted.mapq10.wig ws2001ss501_denominator_L1800469_Scc1_Ty1_wt_soni_Input_1_to_HGAP3_Tb427v9_bwa.sorted.mapq10.wig ws2001ss501 denominator L1800475 Scc1 Ty1 wt soni Input 2 to HGAP3 Tb427v9 bwa.sorted.mapq10.wig ws2001ss501_numerator_L1800343_Scc1_Ty1_wt_soni_ChIP_0_to_HGAP3_Tb427v9_bwa.sorted.mapq10.wig ws2001ss501_numerator_L1800468_Scc1_Ty1_wt_soni_ChIP_1_to_HGAP3_Tb427v9_bwa.sorted.mapq10.wig ws2001ss501_numerator_L1800474_Scc1_Ty1_wt_soni_ChIP_2_to_HGAP3_Tb427v9_bwa.sorted.mapq10.wig ws2001ss501_ratio_L1800344_Scc1_Ty1_wt_soni_Input_0_to_HGAP3_Tb427v9_bwa.sorted.mapq10_vs_L1800343_Scc1_ Ty1_wt_soni_ChIP_0_to_HGAP3_Tb427v9_bwa.sorted.mapq10.wig

ws2001ss501_ratio_L1800469_Scc1_Ty1_wt_soni_Input_1_to_HGAP3_Tb427v9_bwa.sorted.mapq10_vs_L1800468_Scc1_ Ty1_wt_soni_ChIP_1_to_HGAP3_Tb427v9_bwa.sorted.mapq10.wig

ws2001ss501_ratio_L1800475_Scc1_Ty1_wt_soni_Input_2_to_HGAP3_Tb427v9_bwa.sorted.mapq10_vs_L1800474_Scc1_ Ty1_wt_soni_ChIP_2_to_HGAP3_Tb427v9_bwa.sorted.mapq10.wig ws501ss101_denominator_L1800344_Scc1_Ty1_wt_soni_Input_0_to_HGAP3_Tb427v9_bwa.sorted.mapq10.wig ws501ss101_denominator_L1800469_Scc1_Ty1_wt_soni_Input_1_to_HGAP3_Tb427v9_bwa.sorted.mapq10.wig ws501ss101_denominator_L1800475_Scc1_Ty1_wt_soni_Input_2_to_HGAP3_Tb427v9_bwa.sorted.mapq10.wig ws501ss101_numerator_L1800343_Scc1_Ty1_wt_soni_ChIP_0_to_HGAP3_Tb427v9_bwa.sorted.mapq10.wig ws501ss101_numerator_L1800468_Scc1_Ty1_wt_soni_ChIP_1_to_HGAP3_Tb427v9_bwa.sorted.mapq10.wig ws501ss101_numerator_L1800474_Scc1_Ty1_wt_soni_ChIP_2_to_HGAP3_Tb427v9_bwa.sorted.mapq10.wig ws501ss101_ratio_L1800344_Scc1_Ty1_wt_soni_Input_0_to_HGAP3_Tb427v9_bwa.sorted.mapq10_vs_L1800343_Scc1_Ty 1_wt_soni_ChIP_0_to_HGAP3_Tb427v9_bwa.sorted.mapq10.wig

ws501ss101_ratio_L1800469_Scc1_Ty1_wt_soni_Input_1_to_HGAP3_Tb427v9_bwa.sorted.mapq10_vs_L1800468_Scc1_Ty 1_wt_soni_ChIP_1_to_HGAP3_Tb427v9_bwa.sorted.mapq10.wig

ws501ss101_ratio_L1800475_Scc1_Ty1_wt_soni_Input_2_to_HGAP3_Tb427v9_bwa.sorted.mapq10_vs_L1800474_Scc1_Ty 1_wt_soni_ChIP_2_to_HGAP3_Tb427v9_bwa.sorted.mapq10.wig

ChIP Scripts

ChIP_Scc1_Ty_V9_mapping_covernant.sh

MNase_ChIP_H3V_Ty_V9_mapping_covernant.sh

MNase_ChIP_H4V_Ty_V9_mapping_covernant.sh

extract.sh

ratio.sh

run_alignment.sh

csv files (for generation of metaplots)

2018-04-19_cTTS_2.csV

2018-04-19_sTSS_2.csV

Genome browser session (e.g. $\underline{\text { UCSC) }}$

\section{Methodology}

Replicates

Sequencing depth not used

MNase-ChIP-seq experiments were performed in duplicates with an input control for each experiment.

Scc1-ChIP experiments were performed in triplicates with an input control for each experiment.

L1800150_H3 V_Ty_MNase_ChIP_1: NextSeq 500 Mid Output Kit, 150 cycles; total number of paired-end reads: 2840144; read length: 76 bp; paired-end

L1800151_H3_V_Ty_MNase_Input_1: NextSeq 500 Mid Output Kit, 150 cycles; total number of paired-end reads: 4176616; read length: 76 bp; paired-end

L1800152_H3_V_Ty_MNase_ChIP_2: NextSeq 500 Mid Output Kit, 150 cycles; total number of paired-end reads: 3116324; read length: 76 bp; paired-end

L1800153_H3_V_Ty_MNase_Input_2: NextSeq 500 Mid Output Kit, 150 cycles; total number of paired-end reads: 3979824; read length: 76 bp; paired-end

L1800154_H4_V_Ty_MNase_ChIP_1: NextSeq 500 Mid Output Kit, 150 cycles; total number of paired-end reads: 3582319; read length: 76 bp; paired-end

L1800155_H4_V_Ty_MNase_Input_1: NextSeq 500 Mid Output Kit, 150 cycles; total number of paired-end reads: 4636597; read length: 76 bp; paired-end

L1800156_H4_V_Ty_MNase_ChIP_2_R1: NextSeq 500 Mid Output Kit, 150 cycles; total number of paired-end reads: 3924511; read length: 76 bp; paired-end

L1800157_H4_V_Ty_MNase_Input_2_R1: NextSeq 500 Mid Output Kit, 150 cycles; total number of paired-end reads: 4692475; read length: 76 bp; paired-end

L1800343_Scc1_Ty1_wt_soni_ChIP_0: NextSeq 500 Mid Output Kit, 150 cycles; total number of paired-end reads: 9264521; read length: 76 bp; paired-end

L1800344_Scc1_Ty1_wt_soni_Input_0: NextSeq 500 Mid Output Kit, 150 cycles; total number of paired-end reads: 13017782; read length: 76 bp; paired-end

L1800468_Scc1_Ty1_wt_soni_ChIP_1: NextSeq 500 Mid Output Kit, 150 cycles; total number of paired-end reads: 6538932; read length: 76 bp; paired-end 
Antibodies

Peak calling parameters

Data quality

Software
L1800469_Scc1_Ty1_wt_soni_Input_1: NextSeq 500 Mid Output Kit, 150 cycles; total number of paired-end reads: 6910598; read length: 76 bp; paired-end

L1800474_Scc1_Ty1_wt_soni_ChIP_2: NextSeq 500 Mid Output Kit, 150 cycles; total number of paired-end reads: 6370430; read length: 76 bp; paired-end

L1800475_Scc1_Ty1_wt_soni_Input_2: NextSeq 500 Mid Output Kit, 150 cycles; total number of paired-end reads: 5353926; read length: 76 bp; paired-end

All ChIP-seq targets were Ty1-taggged and therefore pulled down with a BB2 Antibody (A novel epitope tag system to study protein targeting and organelle biogenesis in Trypanosoma brucei.

Bastin P, Bagherzadeh Z, Matthews KR, Gull K

Mol Biochem Parasitol. 1996 May; 77(2):235-9.)

For all datasets, sequencing reads were mapped using bwa mem (https://arxiv.org/abs/1303.3997), version 0.7.12-r1039, converted to bam using samtools-1.8 view $-b$, sorted using samtools-1.8 sort and indexed using samtools index. If required, uniquely mapped reads were extracted using samtools view -bq 10. Coverage plots were generated using COVERnant (https://github.com/konrad/COVERnant).

Quality trimming of reads was performed using the Fastx package (version 0.0.1.3). FDR5\% were not determined as no peak calling was perfomed in this study.

Cutadapt (version 1.15); Martin, M. et al. Cutadapt Removes Adapter Sequences From High-Throughput Sequencing Reads. EMBnet.journal 17, 10-12 (2011)

Fastx package (version 0.0.1.3)

COVERnant (version 0.3.2); https://github.com/konrad/COVERnant

Samtools (version 1.8)

BWA-mem (version 0.7.12-r1039); https://arxiv.org/abs/1303.3997

custom shell scripts combining the above tools are available at: Zenodo (DOI 10.5281/zenodo.823671)

\section{Flow Cytometry}

Plots

Confirm that:

Х The axis labels state the marker and fluorochrome used (e.g. CD4-FITC).

Х The axis scales are clearly visible. Include numbers along axes only for bottom left plot of group (a 'group' is an analysis of identical markers).

Х All plots are contour plots with outliers or pseudocolor plots.

Х A numerical value for number of cells or percentage (with statistics) is provided.

\section{Methodology}

Sample preparation

Instrument

Software

Cell population abundance

Gating strategy

Xick this box to confirm that a figure exemplifying the gating strategy is provided in the Supplementary Information.
$1 \times 10^{*} 6$ cells were centrifuged in a chilled microtube at $1,500 \mathrm{~g}$ for $4 \mathrm{~min}$ at $4^{\circ} \mathrm{C}$. Cells were resuspended in 100 ?l of ice cold HMI-11 and a VSG-specific antibody (Anti-VSG-2 or Anti-VSG-13; Figueiredo et al. (2008) was added. After 60 min of incubation at $4{ }^{\circ} \mathrm{C}$ with gentle shaking, cells were washed three times in 500 ?l of ice cold $\mathrm{HMI}-11$, resuspended in 100 ?l of cold $\mathrm{HMI}-11$ and incubated with an Alexa Fluor 488-conjugated secondary antibody for $20 \mathrm{~min}$. The cells were washed twice with 500 ?! of TDB.

\section{BD FACSCalibur type C4A, Becton Dickinson}

CellQuest Pro, Version 6.0

Post-sort fractions were not used in this study

Trypanosomes are very homogenous. Only one population was gated in the SSC/FSC window. None-stained WT cells define the negative control. Stained WT cells define the positive population. 